$$
1 / 2 / .9985(1)
$$

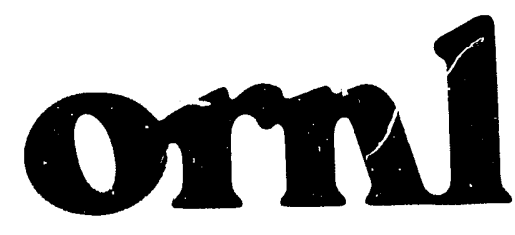

OAK RIDGE NATIONAL

LABORATORY

makitin mapueța

ORNL-6703

\title{
Multifracture of Ceramic Composites
}

\author{
Y. J. Weitsman \\ H. Zhu
}


This report has reen reproduced directly from the best available copy.

Available to DOE and DOE contractors from the Offlice of Scientific and Technical Information, P.O. Box 62, Oak Ridge, TN 37831 ; prices available from (615) 576-8401, FTS 626-8401.

Available to the public from the National Technical Information Service, U.S. Department of Commerce, 5285 Port Royal Rd., Springfield, VA 22161.

This report was prepared as an account of work sponsored by an agency of the United States Government. Neither the United States Government nor any agency thereof, nor any of their employees, makes any warranty, express or implied, or assumes any legal liability or responsibility for the accuracy, completeness, or usefulness of any informetion, apparatus, product, or process disclosed, or represents that its use would not infringe privately owned rights. Feference herein to any specific commercial product, process, or service by trade name, trademark, manufacturer, or otherwise, does not necessarily constilute or imply its endorsement, recommendation, or favoring by the United States Government or any agency thereof. The views and opinions of authors expressed herein do not necessarily state or reflect those of the United States Government or ally agency thereot. 
ORNL --6703

DE92 009630

Engineeting Technology Division

\title{
MULTIFRACTURE OF CERAMIC COMPOSITES
}

\author{
Y. J. Weitsman \\ Oak Ridge National Laboratory/ \\ The University of Tennessee at Knoxville
}

\section{H. Zhu}

The University of Tennessee at Knoxville

DATE PUBLISHED : MARCH 1992

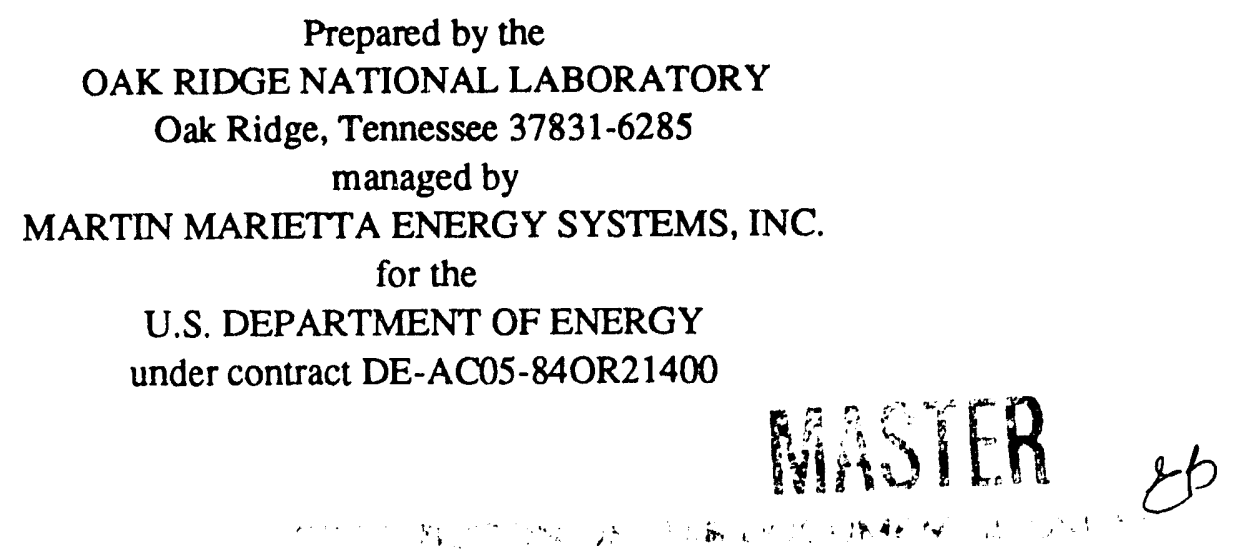




\section{CONTENTS}

LIST OF FIGURES

NOMENCLATURE vii

ABSTRACT 1

1. INTRODUCTION 1

2. FORMULATION OF THE APPROXIMATE MECHANICS SOLUTION 5

3. THE KINEMATICALLY ADMISSIBLE DISPLACEMENT FIELD ............................... 9

3.1 PERFECTLY BONDED INTERFACES (NO SLIP) .......................................... 9

3.2 AN INTERFACIAL SLIP ZONE UNDER CONSTANT SHEAR STRESS ........... 11

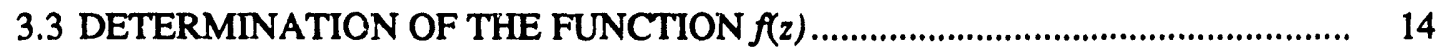

3.4 SELECTION OF THE SHAPE FUNCTIONS $p(r), q(r)$, AND $s(r) \ldots \ldots \ldots \ldots \ldots \ldots \ldots . . . . . . . . . . .17$

4. ANALYYSIS OF MULTIPLE MATRIX CRACKING .............................................. 21

5. STATISTICAL PREDICTIONS OF FIBER FAILURES …................................................ 33

6. SOME CONSIDERATIONS OF FIBER PULL-OUT ENERGY ...................................... 37

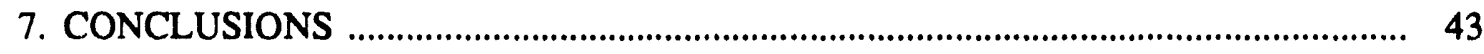

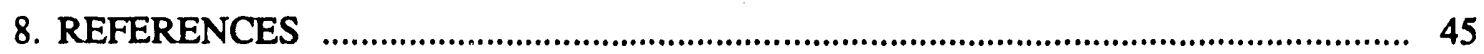

APPENDIX A: FORMULATION OF IN-PLANE FIELDS …............................................ 47

APPENDIX B: THE BOUNDARY ELEMENT SCHEME _................................................. 51 


\section{LIST OF FIGURES}

1.1 The uniaxial stress-strain response of $0^{\circ} \mathrm{SiC} / \mathrm{CAS}$ continuous fiber-reinforced ceramic composites

1.2 Longitudinal and transverse strain against applied tensile load for $0^{\circ}, 43$ vol \% HMU-7740 composites

2.1 The representative volume element

4.1 Two states of matrix fractures in continuous fiber-reinforced ceramic composites

4.2 The effect of interfacial shear strength, $\tau_{s}$, on the stress-strain response of continuous fiber-reinforced ceramic composites (with $\alpha_{f}=\alpha_{m}$ )

4.3 Effects of matrix fracture toughness on stress-strain response of continuous fiber-reinforced ceramic composites $\left(\tau_{s}=0.02, \alpha_{f}=\alpha_{m}\right)$

4.4 Effect of thermal expansions on stress-strain response of continuous fiber-reinforced ceramic composites $\left(\tau_{s}=0.02\right)$

4.5 Stress-strain response at $G_{m}^{c}=5 \mathrm{~N} / \mathrm{m}$ and $\tau_{s}=0.2 \mathrm{GPa}$ for $\alpha_{m}>\alpha_{f}(\longrightarrow)$ and $\alpha_{r n}=\alpha_{f}(-.-)$

4.6 Effect of thermal expansions on stress distribution within fiber (at plane of matrix cracks)

4.7 Depencience of stress distributions within fibers on the interfacial shear strength $\tau_{s}$

4.8 Crack spacing, $L$, in terms of $\log (L / a) / \log (2)$ vs applied stress for (a) $\tau_{s}=0.2 \mathrm{GPa}$, (b) $\tau_{s}=0.02 \mathrm{GPa}$

4.9 Length of slip zone, $l_{s}$, vs applied stress for $\tau_{s}=0.02 \mathrm{GPa}$

5.1 The effect of interfacial shear stress, $\tau_{s}$, (in GPa) on the probability of fiber failure, accounting for radial variation in fiber stress distribution (with $\alpha_{f}=\alpha_{m}$ )

5.2 Probability of fiber failure vs applied stress and its dependence on thermal expansions $\left(\tau_{s}=0.02 \mathrm{GPa}\right)$

5.3 The effect of radial variation of fiber stresses on the probability of fiber failure (a) $\left(\tau_{s}=0.002 \mathrm{GPa}, \alpha_{m}=\alpha_{f}\right)$, (b) $\left(\tau_{s}=0.02 \mathrm{GPa}, \alpha_{m}=\alpha_{f}\right)$

6.1 A sketch of the pull-out mechanism when a matrix crack at $z=L / 2$ is followed by a fiber break at $z=z_{f b}$

6.2 A comparison between $P_{f}$, the probability of fiber failure (solid line), and $P_{f s}$, the probability of fiber failure within a slip zone (dashed line), vs the applied stress $\sigma_{a}$

6.3 The portion of pull-out energy $U_{p}$ [Eq. (53)], nondimensionalized by matrix fracture energy, vs the applied stress, $\sigma_{a}$, for several values of interfacial shear strength, $\tau_{s}$, (in GPa) 
B.1 The Cartesian configuration employed for the boundary elemont method

B.2 Profiles of fiber stresses $\sigma_{z}^{f}(x, z)$ vs $x / a$ computed by the boundary element method (discrete points) compared with $\sigma_{z}^{f}(r, z)$ vs $r / a$ evaluated by the present model 


\section{NOMENCLATURE}

$a$
$A, B, C$
$b$
$E$
$f(z), g(z), k(z), F(z)$
$G$
$i, j$
$L$
$l_{s}$
$P, Q, S$
$p, q, s, \hat{p}, \hat{q}, t$
$P_{f}$
$r, \theta, z$
$T$
$u$
$U$
$V$
$w$
$x, y$
$z_{f b}$
$\alpha$
$\Delta w$
$\varepsilon$
$\varepsilon_{0}$
$\lambda$
$\mathrm{II}_{1}, \mathrm{II}^{*}, \pi_{1}, \pi_{2}$
$\sigma$
$\sigma_{a}$
$\sigma_{0}, M$
$\tau$
$\tau_{s}$
$v$
$\mathfrak{g}_{m}^{c}$

$f$
$m$

radius of fiber

constants associated with various displacements radius of representative volume element

Young modulus shape functions of the coordinate $z$ shear modulus displacement stress and strain indices matrix crack spacing length of slip zone along fiber/matrix interface integrated values of $p, q$, and $s$ shape functions of the coordinate $r$ probability of fiber failure cylindrical coordinates thermal excursion temperature radial displacement

energy

volume fraction axial displacement Cartesian coordinates location of fiber break coefficient of thermal expansion displacement discontinuity at fiber/matrix interface strain average axial strain in test sample Lagrange multiplier energy functionals normal stress average applied axial stress Weibull distribution parameters shear stress maximal shear stress supported by fiber/matrix interface Poisson's ratio critical energy release rate for matrix fracture

\section{SUBSCRIPTS}

fiber

matrix 
MULTIFRACTURE OF CERAMIC COMPOSITES

\author{
Y. J. Weitsman*
}

H. Zhu' ${ }^{\dagger}$

\begin{abstract}
This work presents a mechanistic model for the multifracture process of uniaxially reinforced fibrous ccramic composites under monotonically increasing tension parallel to the fiber direction. The model employs an energy criterion to account for the progression of matrix cracks, bridged by intact fibers, and Weibull failure statistics to relate the failure of the fibers. Consideration is given to the interactions between the foregoing failure processes as well as to the effects of various material parameters on the response of the compcsite.
\end{abstract}

\title{
1. INTRODUCTION
}

Ceramic materials exhibit superior performance at high temperatures, but their utilization in critical structural components is severely curtailed by their excessive brittleness. Techniques to alleviate this brittleness are the subject of intensive research, reviewed by several leadirig investigators in the field (e.g., Schioler and Stiglich 1986, Hillig 1987, and Evans 1990). In one such technique, continuous fiber-reinforced ceramic composites (CFCC) are formed; in these composites, the reinforcing ceramic fibers are typically more ductile than the ceramic matrix. In this case, fracture of the brittle matrix entangles the more compliant fibers in the failure process, resulting in several energy-consuming mechanisms. If appropriately conceived, these mechanisms lead to a gradual structural failure instead of catastrophic collapse.

Typical stress-strain curves are shown in Figs. 1.1 and 1.2. Figure 1.1 exhibits the ductile behavior of unidirectionally reinforced $\mathrm{SiC} / \mathrm{CAS}$ ceramics under uniaxial tension parallel to the fiber direction, with comparison to results obtained by Daniel, Anastassopoulus, and Lee (1989). Figure 1.2, obtained by Nardone and Prewo (1988), shows similar results for HMU7740 composites, with a significant reversal in the lateral strain.

Several striking differences exist between ceramic and, say, polymeric composites. In polymeric composites, the fibers provide stiffness, while the polymer serves essentially as a binder. Consequently, the ratio $E_{f} / E_{m}$ of the fiber and polymer moduli is typically of $O\left(10^{2}\right)$. In addition, to achieve high stiffness it is usually desirable to have strong interfacial bonding

\footnotetext{
*Oak Ridge National Laboratory/The University of Tennessee, Knoxville, Distinguished Scientist in Composite Materials and Structures.

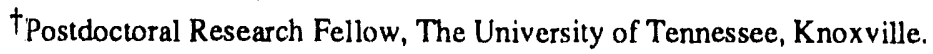




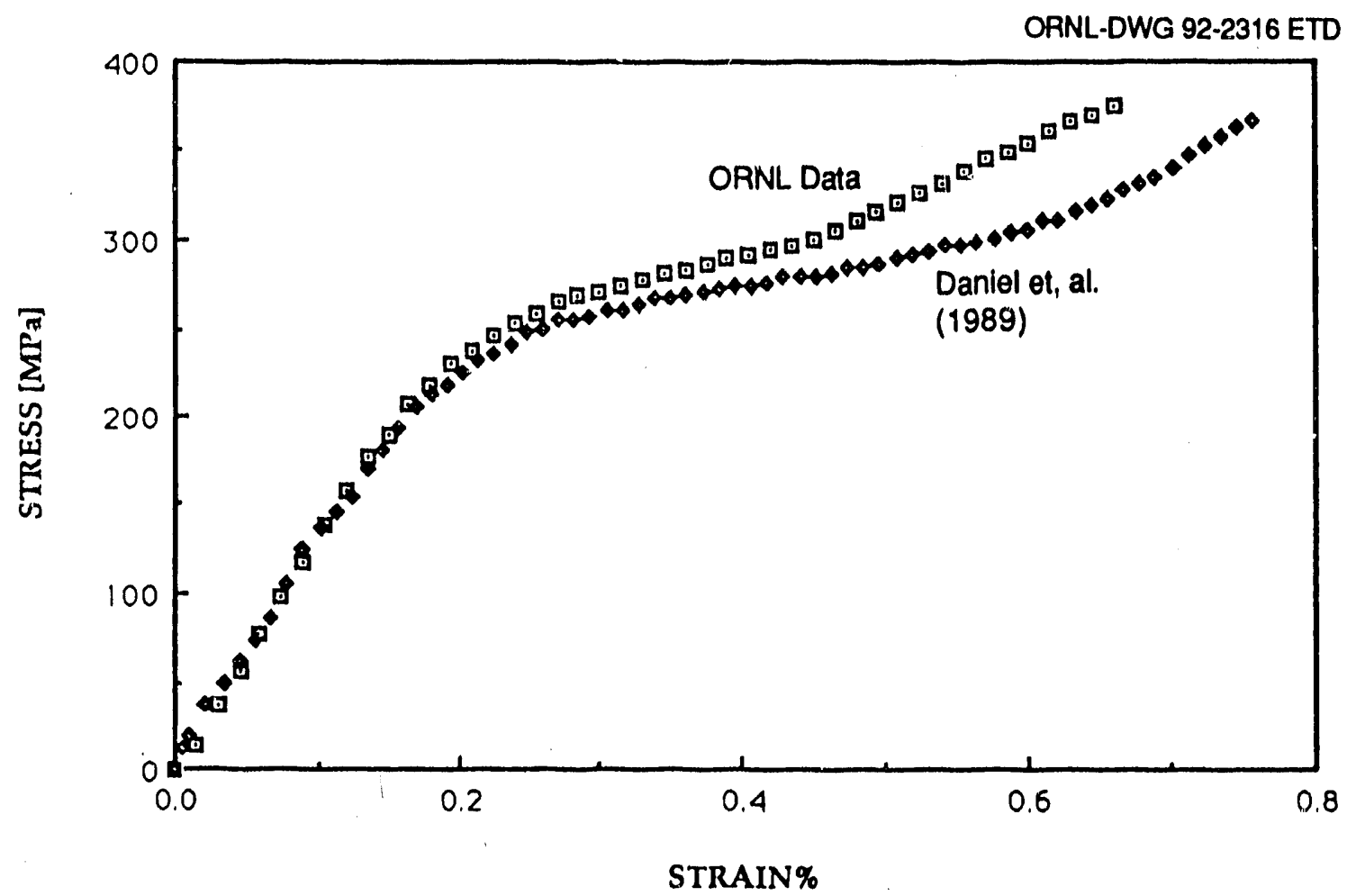

Fig. 1.1. The uniaxial stress-strain response of $0^{\circ} \mathrm{SiC} / \mathrm{CAS}$ continuous fiberreinforced ceramic composites.

between the fiber and matrix materials. In contrast, the primary role of the fibers in ceramic composites is to enhance toughness. Thus, the ratio $E_{f} / E_{m}$ in ceramic composites is typically of $O(1)$. Moreover, it is usually desirable to have weak bonding between the fibers and matrix to enhance interfacial slip, thereby increasing the amount of energy dissipation during the process of matrix cracking. In addition, a weak interfacial bond will deflect matrix cracks around the fibers and bring into play their larger ductility. Therefore, in spite of some similarities, the modelling and analysis of failure of ceramic composites require a different approach than that applied to polymeric composites. Among the several ceramic failure models worth mentioning, we recall those of Aveston, Cooper, and Kelly (1971) and Budiansky, Hutchinson, and Evans (1986). Both works model the fracture behavior of a brittle matrix reinforced by ductile fibers. The former employs a stress criterion for failure, while the latter utilizes energy criteria. Both analyses aim at predicting the onset of the first matrix crack, and both approaches are based upon the shear-lag model.

The latter model assumes that the fibers carry all the tensile load and that the matrix responds in shear only. In addition, the model neglects all shear deformation within the fibers. However, the above assumptions, which are reasonably accurate for $E_{f} / E_{m}$ ratios of $O\left(10^{2}\right)$ as occur in polymeric composites, appear to be deficient in the case of ceramics. It is intuitively obvious that for $E_{f} / E_{m}$ of $\mathrm{O}(1)$, the shears in the fibers and the matrix play equally important roles and that, equivalently, the normal stresses in the matrix should not be neglected. In addition, the normal stresses in the fibers in the vicinity of the matrix crack are expected to 


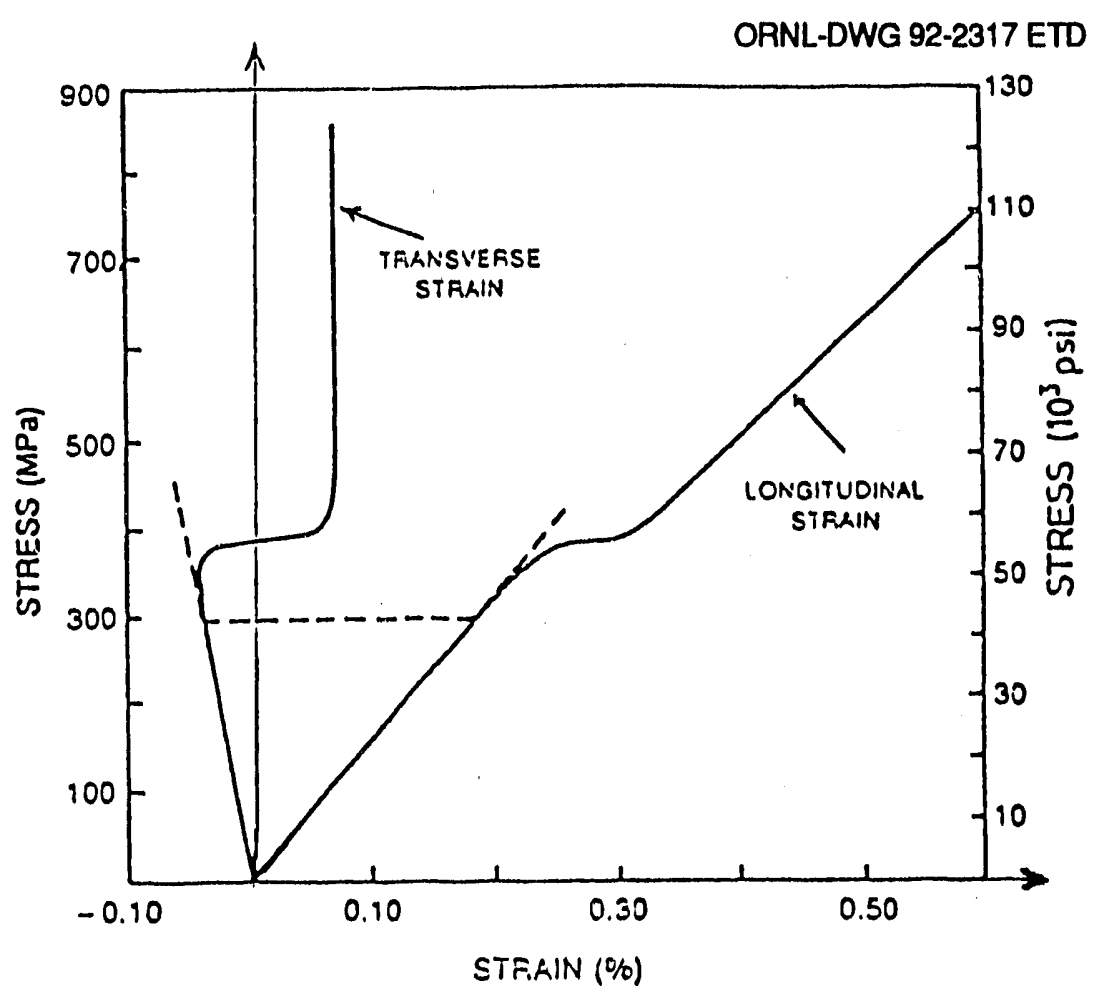

Fig. 1.2. Longitudinal and transverse strain against applied tensile load for $0^{\circ}$, 43 vol \% HMU-7740 composites. Source: V. C. Nardone and K. M. Prewo, 1988, "Tensile Performance of Carbon-Fibre-Reinforced Glass," J. Mater. Sci., 23, 168-180.

exhibit a very pronounced radial variation that plays a critical role in the prediction of fiter failure. The importance of the foregoing stress distribution was alluded to by several investigators (Sutcu 1989, Schwietert and Steif 1990), although they did not quantify or analyze it. Finally, the evolution of multiple cracks within CFCC has not been modelled in the context of energy considerations. A major obstacle in evaluating the energies consumed during the process of progressive failure in CFCC can be traced to yet another inadequacy of the shear-lag model, namely, its limited ability to express the interfacial slip between fibers and matrix and its attendant incapacity to evaluate the dissipative energy.

Energy-based analyses of the multifracture behavior of laminated polymeric composites, when progressive failure occurs at the ply level, were developed by Fang, Schapery, and Weitsman (1986) and by Nuismer and Tan (1988). The latter work employs concepts of shearlag.

An important improvement in the analytical representation of mechanical fields associated with a single matrix crack around a relatively ductile fiber was obtained by McCartney (1989). That representation satisfied most of the field equations and boundary conditions required by an exact solution. Nevertheless, in the present work it was found advantageous to establish another representation more suitable to our purpose of analyzing the interactive effects between fiber failures and the multifracturing process within the matrix. In particular, our representation contains specific details of the stress distribution $\sigma_{z}^{f}(r, z)$ within the fibers, which are 
predicting the ensuing fiber failure process. This aspect seems to be of particular importance because fiber flaws appear to occur more readily near the outer surface of the fiber, thereby corresponding to a Weibull statistical representation that employs surface rather than volume integrals. It turns out that statistical computations employing average values $\bar{\sigma}_{z}^{f}(z)$ predict substantially lower failure probabilities than computations based on the stresses at the interface $r=a$, namely $\sigma_{z}^{f}(a, z)$.

The present formulation assumes that the slip process is caused by relative movement over asperities at the fiber/matrix interfaces. Other considerations, such as Coulomb friction, are obviously possible. These were considered by Dollar and Steif (1988), Steif and Dollar (1988), and, in a broader context, by Hutchinson and Jentsen (1990). 


\section{FORMULATION OF THE APPROXIMATE MECHANICS SOLUTION}

Consider a unidirectionally reinforced composite material, consisting of ceramic fibers and a ceramic matrix, subjected to uniaxial tension parallel to the fiber direction. Beyond a certain level of applied stress, the composite is assumed to develop matrix cracks in planes normal to the fiber direction. These cracks are bridged by intact fibers and are spaced at an average distance $L$. In addition, the composite is assumed to be cooled down by $T$ degrees below its stress-free temperature $T_{0}$.

Let $r, \theta$, and $z$ denote cylindrical coordinates, with corresponding displacements $u, v$, and $w$, respectively; designate stresses by the standard symbols, $\sigma_{r}, \sigma_{z}, \tau_{r z}$, etc. In addition, let $m$ and $f$ denote matrix and fiber properties, respectively.

The thermomechanical problem at hand will be formulated for the representative volume element (RVE) shown in Fig. 2.1. The RVE occupies the volume $0 \leq r \leq b,-L / 2<z<L / 2$, and contains two concentric cylinders. The fiber region is located within $0 \leq r<a,|z| \leq L / 2$, and the matrix domain ranges over $a<r \leq b,|z|<L / 2$. Matrix cracks are supposed to pre-exist at $z=$ $\pm L / 2$, and the interfacial shear stress $\tau_{r z}(a, z)$ is assumed to be limited by a slip stress of magnitude $\tau_{s}$. At $\tau_{r z}(a, z)=\tau_{s}$, relative displacement $\Delta w_{s}$ (slip) is supposed to occur by an amount of $\Delta w_{s}=w_{m}(a, z)-w_{f}(a, z)$, which is yet to be determined. The slip mechanism is assumed to occur as relative motion over interfacial asperities at $r=a$.

We assume that both fiber and matrix materials behave linearly elastic and thai their interfaces remain in lateral contact even in the presence of tangential slip. The maintenance of lateral contact is intuitively justified for materials systems where $\alpha_{m}>\alpha_{f}$ and $v_{m}>v_{f}$, where $\alpha$ and $v$ denote the coefficient of thermal expansion and Poisson's ratio, respectively, and when slip occurs over interfacial asperities of sufficiently large amplitudes.

The boundary value problem for the RVE consists of the linear, isotropic, elastic field equations within the fiber and matrix regions. Employing standard notation, these equations read

where

$$
\varepsilon_{i j}^{f, m}=\frac{1}{\mathrm{E}_{f, m}}\left[\left(1+v_{f, m}\right) \sigma_{i j}^{f, m}-v_{f, m} \sigma_{k k}^{f, m} \delta_{i j}\right]+\alpha_{f, m} T \delta_{i j}
$$

$$
2 \varepsilon_{i j}^{f, m}=u_{i, j}^{f, m}+u_{j, i}^{f, m} .
$$

A.ssuming rotational symmetry, we have $\mathrm{v}=0, \varepsilon_{r \theta}=\varepsilon_{z \theta}=\sigma_{r \theta}=\sigma_{z \theta}=0$, whereby the equilibrium equations read

$$
\begin{gathered}
\frac{\partial \sigma_{z}^{f, m}}{\partial z}+\frac{\partial \tau_{r z}^{f, m}}{\partial r}+\frac{\tau_{r z}^{f, m}}{r}=0, \\
\frac{\sigma_{r}^{f, m}-\sigma_{\theta}^{f, m}}{r}+\frac{\partial \sigma_{r}^{f, m}}{\partial r}+\frac{\partial \tau_{r z}^{f, m}}{\partial z}=0 .
\end{gathered}
$$



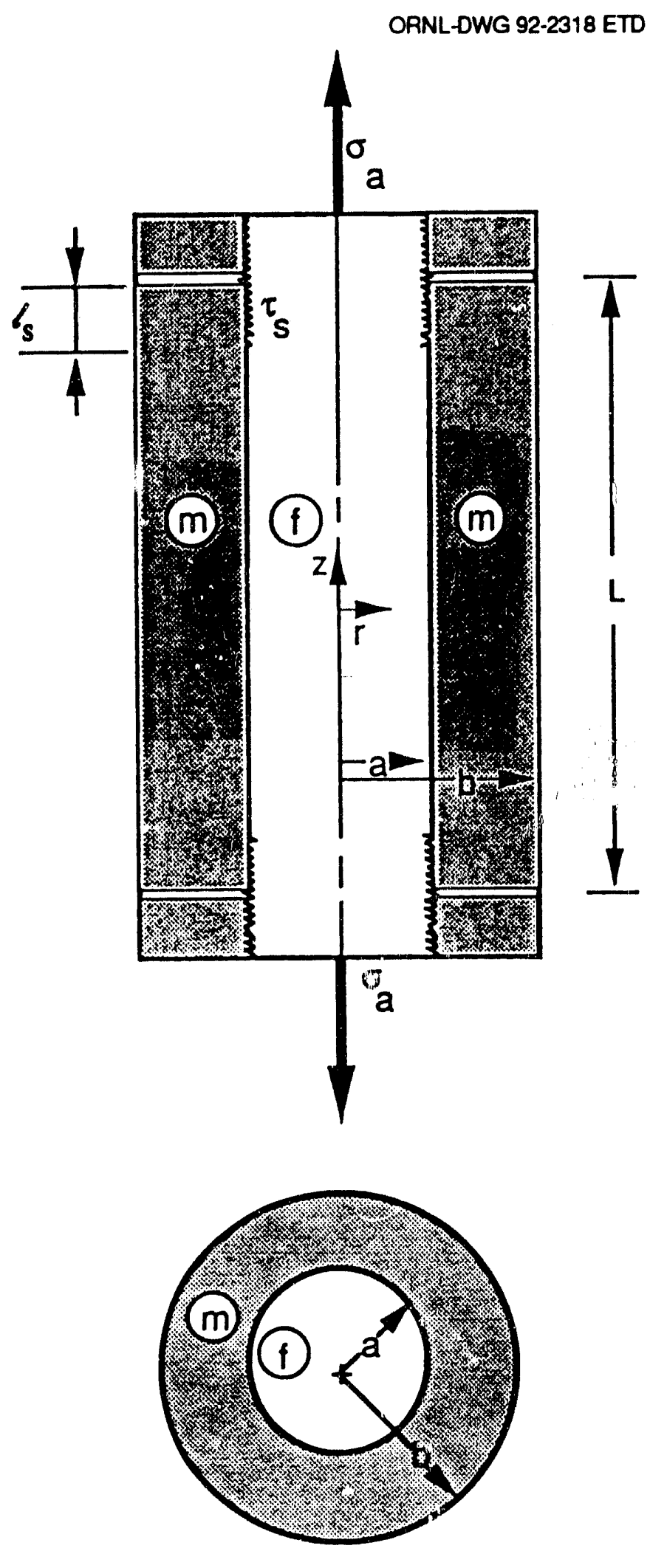

Fig. 2.1. The representative volume element. 
An exact elasticity solution satisfies the following boundary conditions for the RVE:

$$
\left.\begin{array}{c}
\left.\begin{array}{c}
\sigma_{r}^{m}(b, z)=0, \\
\tau_{r z}^{m}(b, z)=0
\end{array}\right\}|z| \leq \frac{L}{2} \\
\sigma_{r}^{m}(a, z)=\sigma_{r}^{f}(a, z), \\
\tau_{r z}^{m}(a, z)=\tau_{r z}^{f}(a, z), \\
u_{m}(a, z)=u_{f}(a, z)
\end{array}\right\}|z| \leq \frac{L}{2}
$$

and

$$
w_{m}(a, z)=w_{f}(a, z)
$$

within the contact region ( $\mathrm{a} a y|z| \leq L / 2-l_{s}$ ),

$$
\left|\tau_{r z}^{m}(a, z)\right|=\left|\tau_{r z}^{f}(a, z)\right|=\tau_{s}
$$

within the slip zone (say $L / 2-l_{s} \leq|z| \leq L / 2$ ).

In addition, the condition of "global" equilibrium gives

$$
2 \pi \int_{0}^{a} \sigma_{z}^{f} r d r+2 \pi \int_{c}^{b} \sigma_{z}^{m} r d r=\pi b^{2} \sigma_{a},
$$

where $\sigma_{a}=\bar{\sigma}_{z}$ is the average value of the applied stress.

The presence of matrix cracks at $z= \pm L / 2$ implies

$$
\left.\begin{array}{l}
\sigma_{z}^{m}\left(r, \pm \frac{L}{2}\right)=0 \\
\tau_{r z}^{m}\left(r, \pm \frac{L}{2}\right)=0
\end{array}\right\} a<r \leq b
$$

Finally, the planes $z= \pm L / 2$ may be viewed as symmetry planes for cylindrical RVEs stacked vertically along the $z$-axis. Therefore,

$$
w_{f}\left(r, \frac{L}{2}\right)=C_{1} \text { and } w_{f}\left(r, \frac{L}{2}\right)=C_{2}, 0 \leq r<a
$$

and

$$
\tau_{r}^{f}\left(r, \pm \frac{L}{2}\right)=0 \quad 0 \leq r<a
$$

In the sequel, an approximate solution to the foregoing boundary value problem will be developed by means of an "enhanced" kinematically admissible field. This field will provide displacements $u_{j}: u_{m}, w_{f}$, and $w_{m}$ that satisfy all the kinematic conditions of our problem, namely, Eqs. (5c), (6a), and (9). 
The paramount stresses in the present problem are $\sigma_{z}^{f, m}$ and $\tau_{r z}^{f, m}$, associated primarily with the displacements $w_{f, m}$ and their spatial derivatives. The in-plane stresses $\sigma_{r}^{f, m}$ and $\sigma_{\theta}^{f, m}$ are mainly due to the lateral contractions $\varepsilon_{r}^{f, m}$ and $\varepsilon_{\theta}^{f, m}$, associated with the displacements $u_{f, m}$. Their effect on $\sigma_{z}^{f, m}$ are of $O\left(v^{2}\right)$, where $v \sim v_{m} \sim v_{f}$ (see Appendix A).

Guided by these observations, we shall construct "enhanced" kinematically admissible fields for $u_{f, m}$ disparately from those for $w_{f, m}$. Specifically, we shall initially consider an uncracked RVE with $u_{f}(r)$ and $u_{m}(r)$ and assume $w_{f}=w_{m}=\varepsilon_{0} z$ [instead of a cracked RVE with $w_{f}(r, z)$ and $\left.w_{m}(r, z)\right]$. For these displacements, we shall employ Eqs. (1) and (2) to generate stresses $\sigma_{r}^{f, m}$ and $\sigma_{\theta}^{f, m}$, which satisfy Eqs. (3b), (4a), (5b), as well as-obviously-(5c). Note that, with the foregoing choice of displacements, $\tau_{r z}^{f}=\tau_{r 2}^{m}=0$. As shown in Appendix A, the uniform strain $\varepsilon_{z}=\varepsilon_{0}$ is related to the applied stress $\sigma_{a}$ and to $T$. In the presence of matrix cracks, we shall employ an iterative procedure to improve the above-mentioned approximation by adjusting the value of $\varepsilon_{0}$ to correspond to an appropriately modified equivalent modulus. The modification will be updated with increasing crack density. We subsequently consider $w_{f}=$ $w_{f}(r, z)$ and $w_{m}=w_{m}(r, z)$ and substitute the already available expressions for $\sigma_{r}^{f, m}$ and $\sigma_{\theta}^{f, m}$ in Eqs. (1) and (2) to relate $\sigma_{z}^{f, m}$ to $\partial w_{f, m} / \partial z$. Then, shear stresses $\tau_{r z}^{f, m}$ that satisfy Eq. (3a) with conditions (4b), (5b), and (6b) are evaluated. For kinematic admissibility, the condition (6a) must of course be satisfied. In addition, we shall also fulfill conditions (7), (8a), and (9).

Clearly, the above kinematically admissible fields $u_{f}(r), u_{m}(r), w_{f}(r, z)$, and $w_{m}(r, z)$ do not provide the exact solution to the boundary value problem stated in Eqs. (1) through (10). The major inadequacy resides in the evaluation of $\tau_{r z}$. The shear stresses $\tau_{r z}^{f, m}$ derived from the equilibrium equation ( $3 a$ ) are inconsistent with expressions deduced from the stress-strain relations (1) and contradict the premise that $\tau_{r z}^{f, m}=0$, which was utilized in the approximate construction of the displacements $u_{f}(r)$ and $u_{m}($. Furthermore, the: ie shear stresses do not satisfy the boundary conditions ( $8 \mathrm{~b}$ ) and (10). These shortcomings are 1ot surprising because an approximate solution cannot handle the extremely intricate comer singularity that, under perfect interfacial contact, occurs along the circle $r=a, z=L / 2$, where the symmetry of the stress tensor no longer holds and $\tau_{z r} \neq \tau_{r z}$ (Bogy 1968). . On the other hand, the inadequacy of our solution is mitigated by the fact that the approximation is optimized through the use of a minimum potential energy principle and guided by a numerical solution to a simpler, but closely related, problem.

\footnotetext{
*However, this singularity may be eliminated when a crack impinges on a frictional interface (e.g., Dollar and Steif 1989).
} 


\section{THE KINEMATICALLY ADMISSIBLE DISPLACEMENT FIELD}

\subsection{PERFECTLY BONDED INTERFACES (NO SLIP)}

It is well known (e.g., Timoshenko and Goodier 1951) that the following radial displacements

$$
\begin{gathered}
u_{f}=A r, \\
u_{m}=A r+B\left(r-\frac{a^{2}}{r}\right),
\end{gathered}
$$

together with $w_{f}=w_{m}=\varepsilon_{0} z$, generate stresses according to Eq. (1) that satisfy the equilibrium equation (3b). In the sequel, expressions (11) represent the kinematically admissible displacements $u_{m}$ and $u_{f}$.

Tuming to the displacements $w_{f}$ and $w_{m}$, introduce first the shape functions $p(r), q(r)$, and $s(r)$ with the following stipulations: $p(0)=q(a)=0, p(a)=q(0)=s(a)=1$. In addition, denote

$$
P=\frac{2}{a^{2}} \int_{0}^{a} r p(r) d r, Q=\frac{2}{a^{2}} \int_{0}^{a} r q(r) d r, S=\frac{2}{b^{2}-a^{2}} \int_{a}^{b} r s(r) d r .
$$

Assume the following forms for the strains $\varepsilon_{2}$ :

$$
\begin{gathered}
\varepsilon_{z}^{f}=p(r)[f(z)+C]+q(r) g(z), \\
\varepsilon_{z}^{m}=s(r) f(z)+C .
\end{gathered}
$$

These strains will generate displacements $w_{f, m}=\int_{0}^{z} \varepsilon_{z}^{f, m}(\hat{z}, r) d \hat{z}$, which are continuous at the interface $r=a$ for all continuous functions $f(z)$ and $g(z)$ and for any arbitrary constant $C$.

In view of Eqs. (1), (11), and (12), we have

$$
\sigma_{z}^{f}=\frac{E_{f}\left(1-v_{f}\right)\{p(r)[f(z)+C]+q(r) g(z)\}}{\left(1+v_{f}\right)\left(1-2 v_{f}\right)}+\frac{2 E_{f} v_{f} A}{\left(1+v_{f}\right)\left(1-2 v_{f}\right)}-\frac{E_{f}}{1-2 v_{f}} \alpha_{f} T
$$

and

$$
\sigma_{z}^{m}(r, z)=\frac{E_{m}\left(1-v_{m}\right)[s(r) f(z)+C]}{\left(1+v_{m}\right)\left(1-2 v_{m}\right)}+\frac{2 E_{m} v_{m}(A+B)}{\left(1+v_{m}\right)\left(1-2 v_{m}\right)}-\frac{E_{m}}{1-2 v_{m}} \alpha_{m} T .
$$

To satisfy boundary condition (8a), the form of Eq. (13b) must be modified to read

$$
\sigma_{z}^{m}(r, z) \sim s(r) f(z) \text { with } f\left( \pm \frac{L}{2}\right)=0 \text {. }
$$

Consequently, we have

$$
C=\frac{1+v_{m}}{1-v_{m}} \alpha_{m} T-\frac{2 v_{m}(A+B)}{1-v_{m}}
$$


and

$$
\sigma_{z}^{m}(r, z)=\frac{E_{m}\left(1-v_{m}\right) s(r) f(z)}{\left(1+v_{m}\right)\left(1-2 v_{m}\right)}
$$

Denote now

$$
E_{f}^{*}=\frac{E_{f}\left(1-v_{f}\right)}{\left(1+v_{f}\right)\left(1-2 v_{f}\right)}, E_{m}^{*}=\frac{E_{m}\left(1-v_{m}\right)}{\left(1+v_{m}\right)\left(1-2 v_{m}\right)}
$$

and

$$
\sigma_{0}=V_{f} E_{f}^{*}\left(C P-\frac{1+v_{f}}{1-v_{f}} \alpha_{f} T+\frac{2 v_{f} A}{1-v_{f}}\right),
$$

with the volume fractions $V_{f}=a^{2} / b^{2}$ and $V_{m}=\left(b^{2}-a^{2}\right) / b^{2}$. Imploying Eq. (7), the force balanc in the $z$-direction gives

$$
\sigma_{a}=V_{f} E_{f}^{*}[P f(z)+Q g(z)]+V_{m} E_{m}^{*} S f(z)+\sigma_{0},
$$

whereby $g(z)$ can be expressed in terms of $f(z)$ as follows

$$
g(z)=\left[\frac{\sigma_{a}-\sigma_{0}}{V_{f} E_{f}^{*}}-P f(z)-\frac{V_{m} E_{m}^{*}}{V_{f} E_{f}^{*}} S f(z)\right] / Q .
$$

Substituting expression (15) in Eqs. (12) and (13a) yields

$$
\begin{aligned}
& \sigma_{z}^{f}=E_{f}^{*} f(z)\left[p(r)-\frac{q(r)}{Q}\left(P+S \frac{V_{m} E_{m}^{*}}{V_{f} E_{f}^{*}}\right)\right]+E_{f}^{*} C[p(r)-P]+\frac{\sigma_{0}}{V_{f}}\left[1-\frac{q(r)}{Q}\right]+\frac{\sigma_{a} q(r)}{V_{f} Q}, \\
& \varepsilon_{z}^{f}=f(z)\left[p(r)-\frac{q(r)}{Q}\left(P+S \frac{V_{m} E_{m}^{*}}{V_{f} E_{f}^{*}}\right)\right]+p(r) C+\frac{\left(\sigma_{a}-\sigma_{0}\right) q(r)}{Q V_{f} E_{f}^{*}} .
\end{aligned}
$$

The latter equation gives

$$
w_{f}=F(z)\left[p(r)-\frac{q(r)}{Q}\left(P+S \frac{V_{m} E_{m}^{*}}{V_{f} E_{f}^{*}}\right)\right]+p(r) C z+\frac{\left(\sigma_{a}-\sigma_{0}\right) q(r) z}{Q V_{f} E_{f}^{*}} .
$$

Employing the equilibrium equation (3a) to determine the shear stress, we have

$$
\tau_{r z}^{f}=-\frac{1}{r} \int_{0}^{r} r^{*} \frac{\partial \sigma_{z}^{f}}{\partial z} d r^{*}=-\frac{E_{f}^{*} f^{\prime}(z)}{r} \int_{0}^{r}\left[p\left(r^{*}\right)-\frac{q\left(r^{*}\right)}{Q}\left(P+S \frac{V_{m} E_{m}^{*}}{V_{f} E_{f}^{*}}\right)\right] r^{*} d r^{*} .
$$

As noted earlier, the result (16d) is inconsistent with the outcome derived from the stress-strain relations (1).

Tuming to the matrix region $a \leq r \leq b,|z|<L / 2$ we obtain

$$
\sigma_{z}^{m}=E_{m}^{*} f(z) s(r)
$$




$$
\begin{gathered}
\varepsilon_{z}^{m}=s(r) f(z)+C, \\
w_{m}=s(r) F(z)+C z,
\end{gathered}
$$

and

$$
\begin{aligned}
\tau_{r z}^{m} & =-\frac{1}{r} \int_{0}^{a} r^{*} \frac{\partial \sigma_{z}^{f}}{\partial z} d r^{*}-\frac{1}{r} \int_{a}^{r} r^{*} \frac{\partial \sigma_{z}^{m}}{\partial z} d r^{*} \\
& =\frac{f^{\prime}(z) E_{m}^{*}}{r}\left[0.5 a^{2} s \frac{V_{m}}{V_{f}}-\int_{a}^{r} s\left(r^{*}\right) r^{*} d r^{*}\right] .
\end{aligned}
$$

In Eqs. (16c) and (17c), we denoted $F(z)=\int_{0}^{z} f\left(z^{*}\right) d z^{*}$.

\subsection{AN INTERFACIAL SLIP ZONE UNDER CONSTANT SHEAR STRESS}

It is assumed that the absolute value of the interfacial shear stress $\left|\tau_{i}(z)\right|=\left|\tau_{r z}(a, z)\right|$ cannot exceed the limiting value of $\tau_{s}$ and that interfacial slip in the amount of $\Delta w(z)=w_{m}(a, z)-$ $w_{f}(a, z)$ occurs when $\tau_{i}(z)= \pm \tau_{s}$. The direction of $\Delta w$ coincides with the sign of $\tau_{i}$. In this circumstance, boundary condition (6b) replaces the displacement continuity condition (6a).

To focus ideas, assume slip to occur at the cylindrical interface $r=a_{n} L / 2-l_{s}<z \leq L / 2$. In this case, we confine the range of applicability of the foregoing expressions for perfectly bonded interfaces to the region $0 \leq z \leq L / 2-l_{s}$ (instead of $0 \leq z \leq L / 2$ ) and construct a "slip zone solution" for the region $0 \leq r \leq b, L / 2-l_{s}<z \leq L / 2$.

The "slip zone formulation" will satisfy the same field equations and boundary conditions as the foregoing formulation for perfectly bonded interfaces, except that Eq. (6b; replaces Eq. (6a). In addition, the two formulations must blend together to ascertain continuity of tractions and displacements at $z=L / 2-l_{s}$, namely, continuity of $u_{m}, u_{f}, w_{m}, w_{f}, \sigma_{i}^{\prime m}, \sigma_{z}^{f}$, and $\tau_{r z}^{m}, \tau_{r z}^{f}$ at $z=L / 2-l_{s}$.

In view of Eqs. (3a) and (6b), we now have

$$
\tau_{r z}^{f}(a, z)=-\frac{1}{a} \int_{0}^{a} r \frac{\partial \sigma_{z}^{f}}{\partial z} d r=-\tau_{s}=\tau_{r z}^{m}(a, z), \quad \frac{L}{2}-l_{s}<z \leq \frac{L}{2} .
$$

Guided by Eq. (19), we select

$$
\sigma_{z}^{f}=\left(\frac{L}{2}-z\right) \hat{p}(r)+\hat{q}(r)+H(r, z)
$$

and

$$
\sigma_{z}^{m}=\left(\frac{L}{2}-z\right) \hat{s}(r)
$$

with the following provisos: 


$$
\begin{gathered}
\frac{1}{a} \int_{0}^{a} r \hat{p}(r) d r=\tau_{s}, \\
\int_{0}^{a} r \frac{\partial H(r, z)}{\partial z} d r=0, \\
\int_{0}^{a} r \hat{p}(r) d r+\int_{a}^{b} r \hat{s}(r) d r=0, \\
\int_{0}^{a} r \hat{q}(r) d r=\sigma_{a} \frac{b^{2}}{2}, \\
\int_{0}^{a} r H(r, z) d r=0,
\end{gathered}
$$

Conditions (21a) and (21b) satisfy Eq. (19), condition (21c) gives $\tau_{r z}^{m}(b, z)=0$, and conditions (21d) and (21e) with (21c) satisfy Eq. (7).

Conditions (21b) and (21e) can be satisfied simultaneously by selecting $H(r, z)=t(r) k(z)$, with $k(z)=\left(z-L / 2+l_{s}\right)^{2}$ and $\int_{0}^{a} r t(r) d r=0$. The foregoing choice of $k(z)$ also facilitates the establishment of traction and displacement continuity at $z=L / 2-l_{s}$. Traction continuity at $z=L / 2-l_{s}$ between the expressions listed in Eqs. (16a), (16d), (17a), and (18) that are consistent with the forms proposed in Eq. (20) can now be established in a straightforward manner. We obtain

$$
\begin{gathered}
\sigma_{z}^{f}=\frac{2 \tau_{s} V_{f} E_{f}^{*}}{a S V_{m} E_{m}^{*}}\left(\frac{L}{2}-z\right)\left[p(r)-\frac{q(r)}{Q}\left(P+S \frac{V_{m} E_{m}^{*}}{V_{f} E_{f}^{*}}\right)\right]+E_{f}^{*} C[p(r)-P] \\
+\frac{\sigma_{0}}{V_{f}}\left[1-\frac{q(r)}{Q}\right]+\frac{\sigma_{a} q(r)}{V_{f} Q}+t(r) k(z), \\
\sigma_{z}^{m}=s(r) \frac{2 \tau_{s} V_{f}}{a S V_{m}}\left(\frac{L}{2}-z\right),
\end{gathered}
$$

and

$$
\begin{gathered}
\tau_{r z}^{f}=\frac{2 \tau_{s} V_{f} E_{f}^{*}}{a S V_{m} E_{m}^{*} r} \int_{0}^{r}\left[p\left(r^{*}\right)-\frac{q\left(r^{*}\right)}{Q}\left(P+S \frac{E_{m}^{*} V_{m}}{E_{f}^{*} V_{f}}\right)\right] r^{*} d r^{*} \\
-\frac{2\left(\mathrm{z}-\mathrm{L} / 2+1_{s}\right)}{\mathrm{r}} \int_{0}^{\mathrm{r}} \mathrm{t}\left(\mathrm{r}^{*}\right) \mathrm{r}^{*} \mathrm{dr} \mathrm{r}^{*} \\
\tau_{r z}^{m}=-\frac{2 \tau_{s}}{a S r}\left[\frac{a^{2} S}{2}-\frac{V_{f}}{V_{m}} \int_{a}^{r} r^{*} s\left(r^{*}\right) d r^{*}\right] .
\end{gathered}
$$

Note that continuity of normal tractions at $z=L / 2-l_{s,} 0 \leq r \leq b$ requires that 


$$
f\left(\frac{L}{2}-l_{s}\right)=\frac{2 \tau_{s} i_{s} V_{f}}{a S V_{m} E_{m}^{*}},
$$

while continuity of shear tractions demands

$$
\frac{\partial f\left(z=L / 2-l_{s}\right)}{\partial z}=-\frac{2 \tau_{s} V_{f}}{a S V_{m} E_{m}^{*}} .
$$

Equations (23a) and (23b) do not contradict each other, because any one of them can be employed to determine the unknown value of $l_{s}$. The remaining equation serves as a consistency condition.

Employing Eq. (22a) with the stress-strain relations (1), we obtain

$$
\begin{aligned}
\varepsilon_{z}^{f} & =\frac{2 \tau_{s} V_{f}}{a S V_{m} E_{m}^{*}}\left(\frac{L}{2}-z\right)\left[p(r)-\frac{q(r)}{Q}\left(P+S \frac{V_{m} E_{m}^{*}}{V_{f} E_{f}^{*}}\right)\right]+p(r) C \\
& +\frac{\left(\sigma_{a}-\sigma_{0}\right) q(r)}{Q V_{f} E_{f}^{*}}+\frac{t(r)\left(z-L / 2+l_{s}\right)^{2}}{E_{f}^{*}},
\end{aligned}
$$

which upon integration with respect to $z$ yields

$$
\begin{aligned}
w_{f} & =\frac{\tau_{s} V_{f}\left[(L-z) z-\left(L / 2+l_{s}\right)\left(L / 2-l_{s}\right)\right]}{a S V_{m} E_{m}^{*}}\left[p(r)-\frac{q(r)}{Q}\left(P+S \frac{V_{m} E_{m}^{*}}{V_{f} E_{f}^{*}}\right)\right] \\
& +F\left(\frac{L}{2}-l_{s}\right)\left[p(r)-\frac{q(r)}{Q}\left(P+S \frac{\left.V_{m} E_{m}^{*}\right)}{V_{f} E_{f}^{*}}\right)\right]+p(r) C z \\
& +\frac{\left(\sigma_{a}-\sigma_{0}\right) q(r) z}{Q V_{f} E_{f}^{*}}+\frac{t(r)\left(z-L / 2+l_{s}\right)^{3}}{3 E_{f}^{*}} .
\end{aligned}
$$

The boundary condition (9a), namely $w_{f}(r, L / 2)=$ constant $(0 \leq r<a)$, can be satisfied by an appropriate selection of $t(r)$. This is accomplished by utilizing Eq. (24) to evaluate the average value of $w_{f}$ at $z=L / 2$, then requiring that

$$
w^{f}\left(r, \frac{L}{2}\right)=\bar{w}^{f}\left(z=\frac{L}{2}\right) \quad(0 \leq r<a) .
$$

The condition (25) yields

$$
\begin{aligned}
t(r) & =\left\{-\left[\frac{\tau_{s} l_{s}^{2} V_{f}}{a S V_{m} E_{m}^{*}}+F\left(\frac{L}{2}-l_{s}\right)\right]\left[p(r)-\frac{q(r)}{Q}\left(P+S \frac{V_{m} E_{m}^{*}}{V_{f} E_{f}^{*}}\right)+S \frac{V_{m} E_{m}^{*}}{V_{f} E_{f}^{*}}\right]\right. \\
& \left.+\frac{L}{2} C[P-p(r)]+\frac{\sigma_{a}-\sigma_{0}}{2 V_{f} E_{f}^{*}}\left[1-\frac{q(r)}{Q}\right] L\right\} \frac{3 E_{f}^{*}}{l_{s}^{3}} .
\end{aligned}
$$


Inspection of Eq. (26) shows that $\int_{0}^{a} r t(r) d r=0$, which satisfies the requirements stated in Eqs. (21b) and (21e).

Tuming to the matrix region $\left(L / 2-l_{s} \leq z \leq L / 2, a<r \leq b\right)$, Eq. (22b) yields

$$
\varepsilon_{z}^{m}=s(r) \frac{2 \tau_{s} V_{f}}{a S V_{m} E_{m}^{*}}\left(\frac{L}{2}-z\right)+C
$$

Whereby, upon integration with respect to $z$ and imposition of continuity of $w_{m}$ with $w_{m}(r, z=$ $L / 2-l_{s}$ ) given in Eq. (17c), we obtain

$$
w_{m}=\frac{\tau_{s} V_{f}\left[(L-z) z-\left(L / 2+l_{s}\right)\left(L / 2-l_{s}\right)\right]}{a S V_{m} E_{m}^{*}} s(r)+s(r) F\left(\frac{L}{2}-l_{s}\right)+C z
$$

Note that, within the slip zone, $w_{m}(a, z) \neq w_{f}(a, z)$.

The radial displacements $u_{f}$ and $u_{m}$ within the slip zone are assumed to coincide with those given in Eq. (11). Consequently, we have within both contact and slip regions, namely for $0 \leq z \leq L / 2$,

$$
\begin{aligned}
& \varepsilon_{\theta}^{f}=\varepsilon_{r}^{f}=A, \quad(0 \leq r<a), \\
& \left.\begin{array}{l}
\varepsilon_{r}^{m}=A+B\left(1+\frac{a^{2}}{r^{2}}\right) \\
\varepsilon_{\theta}^{m}=A+B\left(1-\frac{a^{2}}{r^{2}}\right)
\end{array}\right\} \quad(a<r \leq b) .
\end{aligned}
$$

The thermomechanical stresses that correspond to Eqs. (11) and (28), together with $\varepsilon_{z}^{m}=\varepsilon_{z}^{f}=\varepsilon_{0}$, are evaluated in Appendix A. The constants $A$ and $B$ are also determined in Appendix A, thus establishing the value of $C$ that enters expressions (16), (17), (22), (24), and (27). It can be shown that incorporating the in-plane stresses into this analysis affects the results by an order of $v^{2}$.

Also note that the in-plane stresses $\sigma_{r}^{f, m}$ and $\sigma_{\theta}^{f, m}$ that correspond to the displacements $w_{f}$ and $w_{m}$ prescribed in Eqs. (16c), (17c), (24), and (27) are no longer statically admissible, because those displacements depart from $w_{f}=w_{m}=\varepsilon_{0} z$.

In the forthcoming analysis, we shall employ the minimum potential energy principle to determine $f(z)$ and utilize a numerical solution for analogous mechanical fields in Cartesian geometry to guide our choice of the shape functions $p(r), q(r)$, and $s(r)$.

\subsection{DETERMINATION OF THE FUNCTION $f(z)$}

The field equation that governs the function $f(z)$ will be generated through minimizing the potential energy functional $\Pi(k)$ (Washizu 1975), where the displacements $u: u_{m}, u_{f}, w_{m}$, and $w_{f}$ are given by the kinematically admissible fields listed in Eqs. (11), (16c), (17c), (24), and (27). Employing expressions (1) and (2), we generate the strains $\varepsilon_{r}^{f, m}=\partial u^{f, m} / \partial r$, $\varepsilon_{\theta}^{f, m}=u^{f, m} / r, \varepsilon_{z}^{f, m}=\partial w^{f, m} / \partial z$, and $\gamma_{r z}^{f, m}=\partial w^{f, m} / \partial r$, which correspond to the above 
displacements, and stresses that accord with those strains. Noting that $F(z)=\int_{0}^{z} f(s) d s$ and separating the volume of integration in to the fiber and matrix regions, we write

$$
\begin{aligned}
\Pi\left(F, f, l_{s}\right) & =\int_{0}^{a} \int_{0}^{L / 2} \mathcal{E}_{f} r d r d z+\int_{a}^{b} \int_{0}^{L / 2} \mathcal{E}_{m} r d r d z \\
& -\int_{0}^{a} \sigma_{z}^{f}\left(z=\frac{L}{2}\right) w_{f}\left(z=\frac{L}{2}\right) r d r+\int_{L / 2-l_{s}}^{L / 2}\left[w_{f}(r=a)-w_{m}(r=a)\right] a \tau_{s} d z .
\end{aligned}
$$

The above functional also can be divided among the regions of perfect contact and slip, whereby

$$
\Pi=\int_{0}^{L / 2-l_{s}} \pi_{1}\left[f(z), z, l_{s}\right] d z+\int_{L / 2-l_{s}}^{L / 2} \pi_{2}\left[F\left(\frac{L}{2}-l_{s}\right), z, l_{s}\right] d z
$$

where

$$
\pi_{1}=\int_{0}^{a}\left[E_{f}-\sigma_{z}^{f}\left(z=\frac{L}{2}\right) \varepsilon_{z}^{f}\right] r d r+\int_{a}^{b} \mathcal{E}_{m} r d r, \quad 0 \leq z \leq \frac{L}{2}-l_{s}
$$

while

$$
\begin{aligned}
\pi_{2} & =\int_{0}^{a}\left[\mathcal{E}_{f}-\sigma_{z}^{f}\left(z=\frac{L}{2}\right) \varepsilon_{z}^{f}\right] r d r+\int_{a}^{b} \mathcal{E}_{m} r d r \\
& +\frac{t(r=a)\left(z-L / 2+l_{s}\right)^{4} \tau_{s}}{4 l_{s}^{3} E_{f}^{*}}, \quad \frac{L}{2}-l_{s} \leq z \leq \frac{L}{2} .
\end{aligned}
$$

In Eqs. (30), (31), and (32),

$$
\begin{aligned}
\mathcal{E}_{f} & =0.5 E_{f}^{*}\left[\left(\varepsilon_{z}^{f}\right)^{2}+\left(\varepsilon_{r}^{f}\right)^{2}+\left(\varepsilon_{\theta}^{f}\right)^{2}\right]+0.5 \mu_{f}\left(\gamma_{r z}^{f}\right)^{2}+\frac{E_{f}^{*} v_{f}}{1-v_{f}}\left(\varepsilon_{z}^{f} \varepsilon_{r}^{f}+\varepsilon_{r}^{f} \varepsilon_{\theta}^{f}+\varepsilon_{z}^{f} \varepsilon_{\theta}^{f}\right) \\
& -\frac{E_{f}^{*}\left(1+v_{f}\right)}{1-v_{f}} \alpha_{f} \Delta T\left(\varepsilon_{z}^{f}+\varepsilon_{r}^{f}+\varepsilon_{\theta}^{f}\right)
\end{aligned}
$$

and

$$
\begin{aligned}
\mathcal{E}_{m} & =0.5 E_{m}^{*}\left[\left(\varepsilon_{z}^{m}\right)^{2}+\left(\varepsilon_{r}^{m}\right)^{2}+\left(\varepsilon_{\theta}^{m}\right)^{2}\right]+0.5 \mu_{m}\left(\gamma_{r z}^{m}\right)^{2}+\frac{E_{m}^{*} v_{m}}{1-v_{m}}\left(\varepsilon_{z}^{m} \varepsilon_{r}^{m}+\varepsilon_{r}^{m} \varepsilon_{\theta}^{m}+\varepsilon_{z}^{m} \varepsilon_{\theta}^{m}\right) \\
& -\frac{E_{m}^{*}\left(1+v_{m}\right)}{1-v_{m}} \alpha_{m} \Delta T\left(\varepsilon_{z}^{m}+\varepsilon_{r}^{m}+\varepsilon_{\theta}^{m}\right) .
\end{aligned}
$$

Note, however, that the continuity conditions $(23 a, b)$ impose constraints on $\Pi$. To comply with those constraints, construct the modified functional $\Pi^{*}$ expressed by

$$
\begin{aligned}
\Pi^{*} & =\Pi+\lambda_{1}\left[f\left(\frac{L}{2}-l_{s}\right)-\frac{2 \tau_{s} l_{s} V_{f}}{a S V_{m} E_{m}^{*}}\right] \\
& +\lambda_{2}\left[\frac{\partial f\left(z=L / 2-l_{s}\right)}{\partial z}+\frac{2 \tau_{s} V_{f}}{a S V_{m} E_{m}^{*}}\right],
\end{aligned}
$$

where $\lambda_{1}, \lambda_{2}$ are Lagrange multipliers. 
Evaluate now the variation $\delta \Pi^{*}$ and set $\delta \Pi^{*}=0$ for a stationary value of $\Pi^{*}\left[f(z), l_{s}, \lambda_{1}\right.$, $\lambda_{2}$ ]. Performing the differentiations and integrations included in Eqs. (31) and (32), substituting the results in Eq. (30), and consideririg the independent variations $\delta F, \delta l_{s}, \delta \lambda_{1}$, and $\delta \lambda_{2}$ of $\Pi^{*}$, we obtain

$$
\begin{aligned}
\delta \Pi^{*}\left(f, l_{s}, \lambda_{1}, \lambda_{2}\right)= & \int_{0}^{L / 2}-l_{s}\left\{\left[\frac{\partial \pi_{1}}{\partial F(z)}-\frac{\partial^{2} \pi_{1}}{\partial f(z) \partial z}\right] \delta F(z)+\frac{\partial \pi_{1}}{\partial l_{s}} \delta l_{s}\right\} d z \\
& +\frac{\partial \pi_{1}\left(z=L / 2-l_{s}\right)}{\partial F} \delta F\left(\frac{L}{2}-l_{s}\right)-\frac{\partial \pi_{1}(z=0)}{\partial F} \delta F(0) \\
& -\left[\pi_{1}\left(z=\frac{L}{2}-l_{s}\right)-\pi_{2}\left(z=\frac{L}{2}-l_{s}\right)\right] \delta l_{s} \\
& +\int_{L / 2}^{L / 2}-l_{s}\left[\frac{\partial \pi_{2}}{\partial F\left(L / 2-l_{s}\right)} \delta F\left(\frac{L}{2}-l_{s}\right)+\frac{\partial \pi_{2}}{\partial l_{s}} \delta l_{s}\right] d z \\
& -\lambda_{1}\left[\frac{\partial f\left(z=L / 2-l_{s}\right)}{\partial z}+\frac{\left.2 \tau_{s} V_{f}\right] \delta l_{s}}{a S V_{m} E_{m}^{*}}\right]+\left[f\left(\frac{L}{2}-l_{s}\right)-\frac{2 \tau_{s} l_{s} V_{f}}{a S V_{m} E_{m}^{*}}\right] \delta \lambda_{1}-\lambda_{2}\left[\frac{\partial f^{2}\left(z=L / 2-l_{s}\right)}{\partial z^{2}}\right] \delta l_{s} \\
& +\left[\frac{\partial f\left(z=L / 2-l_{s}\right)}{\partial z}+\frac{2 \tau_{s} V_{f}}{a S V_{m} E_{m}^{*}}\right] \delta \lambda_{2} .
\end{aligned}
$$

Collecting terms that correspond to the independent variations $\delta f, \ldots, \delta \lambda_{2}$, the governing equation for $f(z)$ reads

$$
\begin{aligned}
& \frac{\partial f^{2}(z)}{\partial z^{2}}-\left(\beta_{f} / a\right)^{2} f(z)+f_{0}\left(\beta_{f} / a\right)^{2}=0, \\
& 0 \leq z \leq \frac{L}{2}-l_{s},
\end{aligned}
$$

with the boundary conditions (23a) and (2.3b) restated as

$$
\begin{gathered}
f\left(\frac{L}{2}-l_{s}\right)=\frac{2 \tau_{s} l_{s} V_{f}}{a S V_{m} E_{m}^{*}}, \\
\frac{\partial f\left(z=L / 2-l_{s}\right)}{\partial z}=-\frac{2 \tau_{s} V_{f}}{a S V_{m} E_{m}^{*}} .
\end{gathered}
$$

Assuming $w_{f}$ and $w_{m}$ odd in $z$ [whereby $f(z)$ is even in $z$ ], the solution of Eq. (35) with Eq. (36a) reads 


$$
f(z)=f_{0}-\left(f_{0}-\frac{2 \tau_{s} l_{s} V_{f}}{a S V_{m} E_{m}^{*}}\right) \frac{\cosh \left(\beta_{f} z / a\right)}{\cosh \left[\beta_{f}\left(0.5 L-l_{s}\right) / a\right]} .
$$

The condition (36b) provides the expression that determines the length $l_{s}$ of the slip zone

$$
\beta_{f}\left(\frac{f_{0}}{a}-\frac{2 \tau_{s} l_{s} V_{f}}{a^{2} S V_{m} E_{m}^{*}}\right) \frac{\sinh \left[\beta_{f}\left(0.5 L-l_{s}\right) / a\right]}{\cosh \left[\beta_{f}\left(0.5 L-l_{s}\right) / a\right]}=\frac{2 \tau_{s} V_{f}}{a S V_{m} E_{m}^{*}}
$$

In performing the manipulations that lead to Eqs. (34), (35), and (37), we obtained

$$
\beta_{f}=\left(\frac{C_{1}+C_{2}}{C_{3}+C_{4}}\right)^{0.5} \text {, }
$$

where

$$
\begin{aligned}
& C_{1}=\mu_{f} \int_{0}^{a}\left[p^{\prime}(r)-\frac{q^{\prime}(r)}{Q}\left(P+S \frac{V_{m} E_{m}^{*}}{V_{f} E_{f}^{*}}\right)\right]^{2} r d r, \\
& C_{2}=\mu_{m} \int_{a}^{b}\left[s^{\prime}(r)\right]^{2} r d r, \\
& C_{3}=\frac{E_{f}^{*}}{a^{2}} \int_{0}^{a}\left[p(r)-\frac{q(r)}{Q}\left(P+S \frac{V_{m} E_{m}^{*}}{V_{f} E_{f}^{*}}\right)\right]^{2} r d r, \\
& C_{4}=\frac{E_{m}^{*}}{a^{2}} \int_{a}^{b}[s(r)]^{2} r d r, \\
& f_{0}=\frac{-\mu_{f}}{\left(C_{1}+C_{2}\right)} \int_{0}^{a}\left[p^{\prime}(r)-\frac{q^{\prime}(r)}{Q}\left(P+S \frac{V_{m} E_{m}^{*}}{V_{f} E_{f}^{*}}\right)\right]\left[p(r) C+\frac{\left(\sigma_{a}-\sigma_{0}\right) q(r)}{Q V_{f} E_{f}^{*}}\right] r d r .
\end{aligned}
$$

At this stage, it may be noted that adding a function $G(r, z)$, say, to $\sigma_{z}^{m}$ in Eq. (20b), analogous to $H(r, z)$ in Eq. (20a), would lead to the requirements that $G\left(r, z=L / 2-l_{s}\right)=$ $\left[\partial G\left(r, z=L / 2-l_{s}\right)\right] / \partial z=0, \int_{a}^{b} r^{*} G\left(r^{*}, z\right) d r=0$, as well as $G(r, z=L)=0$. Upon incorporating $G(r, z)$ in the variational functional $\Pi^{*}$, the resulting Euler's equation on $G$, together with the above boundary conditions, would yield $G(r, z) \equiv 0$. Consequently, the choice of $\sigma_{z}^{m}$ in Eq. (20b) is consistent with the remainder of the present formulation.

\subsection{SELECTION OF THE SHAPE FUNCTIONS $p(r), q(r)$, AND $s(r)$}

The selection of the shape functions $p(r), q(r)$ and $s(r)$ is guided by numerical results, obtained by means of the boundary element method, to a circumstance similar to the boundary value problem stated in Eqs. (1) through (10), except that the RVE is defined in Cartesian coordinates, namely $-L / 2<z<L / 2$ with "fiber" and matrix regions extending over $|x|<a$ and $a$ $<|x| \leq b$, respectively. The scheme and results are presented in Appendix B. In addition, the 
choice of the above functions is influenced by analytical results obtained recently (Wijeyewickrema et al. 1990) to the problem of a single fiber contained within a concentrically cylindrical region of a multicracked matrix, but in the absence of interfacial slip. Accordingly, we choose

$$
\begin{aligned}
& p(r)=\left(\frac{r}{a}\right)^{n}, \\
& q(r)=1-\left(\frac{r}{a}\right)^{n},
\end{aligned}
$$

and

$$
s(r)=1-D_{1}\left(1-\frac{a}{r}\right)
$$

The unknowns at this stage are $l_{s}, D_{1}$, and $n$, as well as $A$ and $B$ in Eqs. (A.1) and (A.2) (see Appendix $A$ ). These five unknowns can be determined by iteration employing Eqs. (A.18a, b), (A.9), (23a), or (23b) and the requirement that $\partial \sigma_{z} / \partial_{z}=0$ at $z=L / 2, r=0$. The latter condition derives from the observation that $z=L / 2$ is a symmetry plane in the present boundary value problem and should hold in addition to $\tau_{r 2}(0, L / 2)=0$. In an exact solution, we would have $\partial \sigma_{z} / \partial z=0, \tau_{r z}=0$ at $z=L / 2$ and $0 \leq r<a$. However, the approximate character of our solution restricts our ability to satisfy the stated symmetry condition over the full range of $r$.

We have

$$
\frac{\partial \sigma_{z}^{f}}{\partial z}\left(0, \frac{L}{2}\right)=D_{2}+D_{3}=0
$$

where

$$
\begin{aligned}
& D_{2}=\frac{2 \tau_{s} V_{f} E_{f}^{*}}{a n S V_{m} E_{m}^{*}}\left(1+S \frac{V_{m} E_{m}^{*}}{V_{f} E_{f}^{*}}\right), \\
& D_{3}=\frac{6 E_{f}}{n l_{s}^{2}}\left\{\left(1+S \frac{V_{m} E_{m}^{*}}{V_{f} E_{f}^{*}}\right)\left[\frac{\tau_{s} l_{s}^{2} V_{f}}{a S V_{m} E_{m}^{*}}+F\left(\frac{L}{2}-l_{s}\right)\right]+\frac{L}{2}\left(\frac{\sigma_{a}-\sigma_{0}}{V_{f} E_{f}^{*}}-C\right)\right\} .
\end{aligned}
$$

To achieve computational efficiency, the iteration scheme assumes sequential values for the group of the unknowns $\left(A, B, D_{1}\right)$ and subsequently for the pair $\left(n, l_{s}\right)$. For example, for a given $\sigma_{a}$, we start with the uncracked state, whereby $D_{1}=0$ and $A$ and $B$ are determined from Eqs. (A.18a) and (A.18b). These values are then used for a given crack spacing $L$ to solve simultaneously for $n$ and $l_{\mathrm{S}}$ in Eqs. (23b) and (40). At this stage, we compute $\varepsilon_{0}=\bar{\varepsilon}_{z}=\frac{2}{b^{2} L} \int_{0}^{b} \int_{-L / 2}^{L / 2} \varepsilon_{z}(r, z) r d r d z$ and analogous values for $\bar{\sigma}_{z}^{f}$ and $\bar{\sigma}_{z}^{m}$, redefining new $E_{f}$ and $E_{m}$ in Eqs. (A.18a) and (A.18b) as $E_{f}=\bar{\sigma}_{z}^{f} / \bar{\varepsilon}_{z}$ and $E_{m}=\bar{\sigma}_{z}^{m} / \bar{\varepsilon}_{z}$ and recomputing $A$ and $B$. The value of $D_{1}$ is determined by requiring that $\bar{\sigma}_{z}^{m}$ computed with $s(r)$, according to 
Eq. (39c), agrees with $\bar{\sigma}_{z}^{m}$ evaluated from Eq. (A.9). The iteration proceeds until attaining desired accuracy. For cracks spaced at distances $L / 2$, it is possible to start with values of $A, B$, and $D_{1}$, which correspond to the spacing $L$. 


\section{ANALYSIS OF MULTIPLE MATRIX CRACKING}

Consider a fiber-reinforced ceramic composite, subjected to an applied stress $\sigma_{a}$, with planar matrix cracks spaced at distances $L$ apart. Let new planar cracks propagate quasistatically in a straight front in the $x$-direction midway between the existing cracks, namely at spacings of $L / 2$ apart, as shown in Fig. 4.1.

Assume that the volume of the fiber-reinforced composite is filled completely by the RVEs, as shown in Fig. 2.1. In this case, the criterion for the formation of new matrix cracks can be derived by comparing energy levels in "upstream" and "downstream" RVEs. The upstream RVE has cracks spaced at distances $L / 2$, which we denote by "State II," while the downstream element has cracks spaced $L$ apart, designated by "State I."

Let $g_{m}^{c}$ be the critical energy release rate associated with matrix cracking, and let $U$ denote strain-energy density; then, in analogy with Budiansky, Hutchinson, and Evans (1986), the transition from State I to State II is governed, formally, by the criterion

$$
\int_{-L / 2}^{L / 2} \int_{0}^{b}\left[U_{\Pi}(r, z)-U_{\mathrm{I}}(r, z)\right] r d r d z=\pi\left(b^{2}-a^{2}\right) \mathfrak{g}_{m}^{c}
$$

Let $V$ and $S$ denote the volume and outer surface of the RVE, and let $t_{i}$ be the tractions on $S$. Employing the divergence theorem, we have

$$
\int_{V}\left(U_{\mathrm{II}}-U_{\mathrm{I}}\right) d V=\frac{1}{2} \int_{V}\left(\sigma_{i j}^{\Pi} \varepsilon_{i j}^{\Pi}-\sigma_{i j}^{\mathrm{I}} \varepsilon_{i j}^{\mathrm{I}}\right) d V=\frac{1}{2} \int_{S}\left(t_{i}^{\mathrm{I}} u_{i}^{\mathrm{I}}-t_{i}^{\mathrm{I}} u_{i}^{\mathrm{I}}\right) d S
$$

Splitting the left side of Eq. (41) between the fiber and matrix regions, we have

$$
\frac{1}{2} \int_{S_{f}}\left(t_{i f}^{\mathrm{I}} u_{i f}^{\mathrm{I}}-t_{i f}^{\mathrm{I}} u_{i f}^{\mathrm{I}}\right) d S_{f}+\frac{1}{2} \int_{S_{m}}\left(t_{i m}^{\mathrm{I}} u_{i m}^{\mathrm{I}}-t_{i m}^{\mathrm{I}} u_{i m}^{\mathrm{I}}\right) d S_{m}=\pi\left(b^{2}-a^{2}\right) \boldsymbol{g}_{m}^{c}
$$

In State I, the surface $S_{f}$ consists of the horizontal circular regions at $z= \pm L / 2$ and the cylindrical boundary $r=a,|z| \leq L / 2$, which consists of slip zones $r=a, L / 2-l_{s}<|z|<L /$, and a contact region $|z| \leq L / 2-l_{\mathrm{s}}$. In State II, the cylindrical boundary of $S_{f}$ contains a new slip zorie at $r=a,|z|<l_{s}$, whereby the contact region reduces to $l_{s} \leq|z| \leq L / 2-l_{s}$. Similarly, in State I, the surface $S_{m}$ consists of traction-free crack surfaces at $z= \pm L / 2, a<r<b$, a cylindrical boundary $r=a,|z| \leq L / 2$, of common features to $S_{f}$, and a traction-free boundary at $r=b,|z| \leq L / 2$. In State II, the boundary $S_{m}$ contains a new traction-free crack at $z=0, a<r<b$, as well as a new slip zone at $r=a,|z|<l_{s}$, commensurate with $S_{f}$

Consequently, Eq. (42) reduces to the following expression:

$$
\pi b^{2} \sigma_{a}\left[w_{f}^{\mathrm{II}}\left(z=\frac{L}{2}\right)-w_{f}^{\mathrm{I}}\left(z=\frac{L}{2}\right)\right]-U_{\text {slip }}=\pi\left(b^{2}-a^{2}\right) \mathfrak{g}_{m}^{c}
$$

where

$$
U_{\text {slip }}=2 \pi a \tau_{s}\left\{\int_{L / 2-l_{s}}^{L / 2}\left[\Delta w^{\mathbb{I}}(a, z)-\Delta w^{\mathbb{I}}(a, z)\right] d z+\int_{0}^{l_{s}} \Delta w^{\Pi}(a, z) d z\right\}
$$




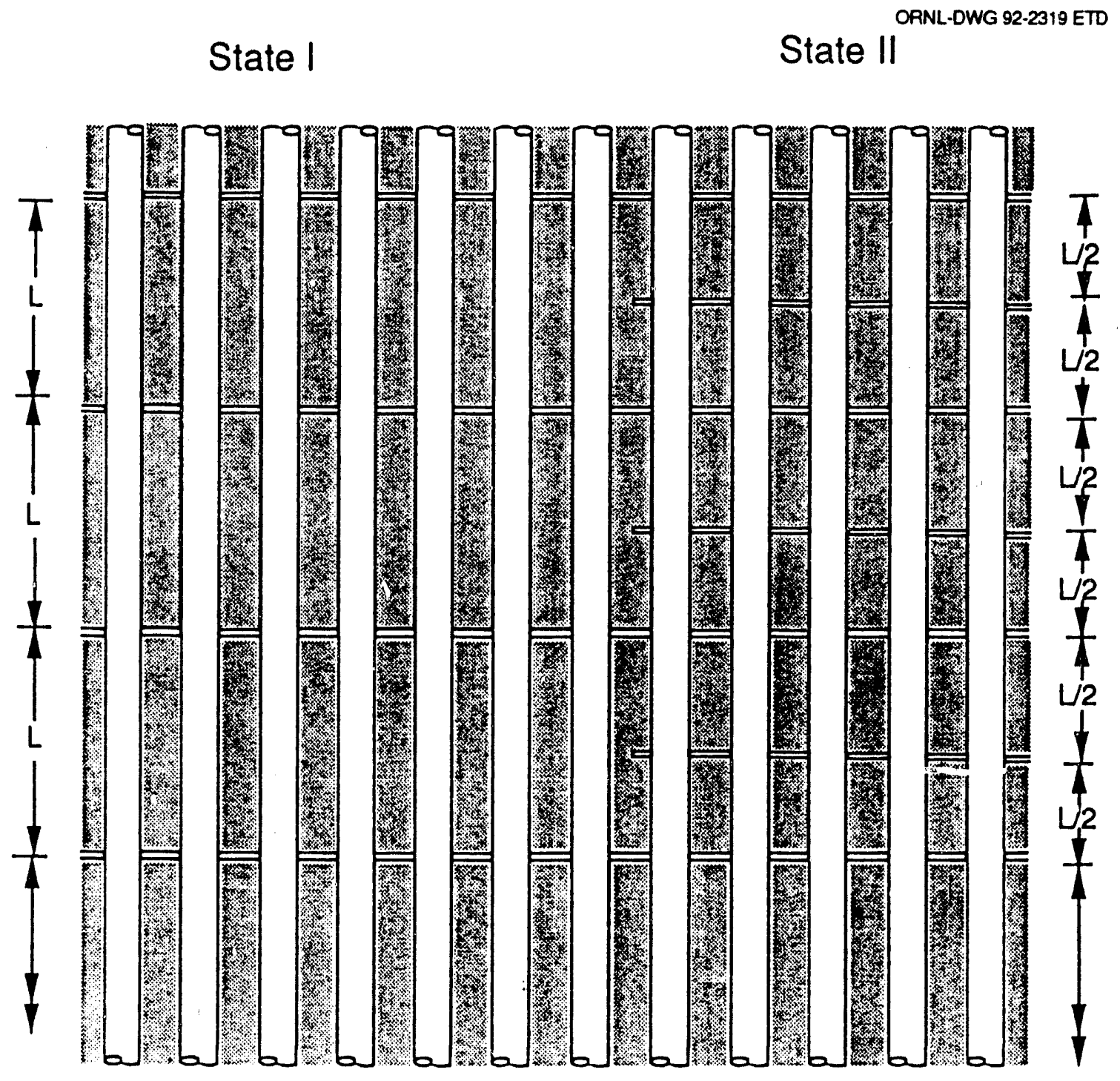

Fig. 4.1. Two states of matrix fractures in continuous fiber-reinforced ceramic composites. State I: Cracks spaced at distance $L$. State II: Cracks spaced at distance $\boldsymbol{L} \mathbf{2}$.

where $\Delta w(a, z)=w_{m}(a, z)-w_{f}(a, z)$, as defined earlier in this work.

Equation (43) can be viewed as a criterion to determine the magnitude of $\sigma_{a}^{c}$ associated with transition from State I to State II. Analogous transitions occur from "state $(N+1)$ " to "state $(N+$ 2)," $N=1,2, \ldots$, when average crack spacing increases from $\left(L / 2^{N}\right)$ to $\left(L / 2^{N+1}\right)$ with applied stress value $\left(\sigma_{a}^{c}\right)_{N}$. Between transitions, namely for $\left(\sigma_{a}^{c}\right)_{N}<\sigma_{a}<\left(\sigma_{a}^{c}\right)_{N+1}$, and under monotonically increasing $\sigma_{a}$, the composite sustains monotonic, continuously increasing strains.

The formal criterion (41) and the attendant results expressed in Eqs. (43) and (44) are subjected to the thermodynamic requirements of irreversibility of dissipation. In the present case, this requirement implies that $U_{\text {slip }}$ must be a nondecreasing function of the applied stress $\sigma_{a}$. Specifically, under monotonically increasing $\sigma_{a}$ as well as during transitions between fracture 
states, the lengths $l_{s}$ of the slip zones cannot decrease in size. Consequently, our computational solution to Eq. (43) included the requirement that the lengths $l_{s}$ of the slip zones associated with State I (say) at $\sigma_{a}=\sigma_{a}^{c}-\varepsilon$ do not decrease upon the transition to State II (say) at $\sigma_{a}=\sigma_{a}^{c}-\varepsilon$. $^{*}$

In the computations of the results exhibited below, we employed the following values for the thermomechanical properties of the fibers and the matrix (unless stated otherwise): $E_{f}=$ $200 \mathrm{GPa}, E_{m}=85 \mathrm{GPa}, v_{f}=0.1, v_{m}=0.1, v_{f}=0.4, a=8.0 \times 10^{-6} \mathrm{~m}, \mathfrak{g}_{m}^{c}=44 \mathrm{~N} / \mathrm{m}$, and $T=$ $-550^{\circ} \mathrm{C}$. Also, when considering $\alpha_{f}>\alpha_{m}$, we took $\alpha_{f}=6.0 \times 10^{-6} /{ }^{\circ} \mathrm{C}$, and $\alpha_{m}=3.0 \times 10^{-6} /{ }^{\circ} \mathrm{C}$, while for $\alpha_{m}>\alpha_{f}$ we employed $\alpha_{f}=3.0 \times 10^{-6 /} \mathrm{C}$, and $\alpha_{m}=6.0 \times 10^{-6} / \mathrm{C}$. These values correspond to SiC/LAS ceramic composites.

Various values were selected for the $\tau_{s}$ and $\mathfrak{g}_{m}^{c}$ to demonstrate their influence on the stressstrain response of the composite material. Furthermore, we chose (somewhat arbitrarily) the spacing $L \cong 30,000 a$ as the initial distance between adjacent cracks to lead off the computations of the stress-strain curves. For the specific examples considered herein, the earliest noticeable departure from linearity occurred at $L \sim 128 a$ and larger values of $L$ yielded stress-strain curves indistinguishable from those shown below. ${ }^{\dagger}$ Results are exhibited in Figs. 4.2 through 4.9.

The predicted stress-strain curves, with longitudinal and transverse strains denoted by $\varepsilon_{L}$ and $\varepsilon_{T}$, are shown in Figs. 4.2 through 4.5. The influence of $\tau_{s}$ is exhibited in Fig. 4.2 (where $\alpha_{f}=$ $\alpha_{m}$ ), the effect of $g_{m}^{c}$ is shown in Fig. 4.3 (with $\tau_{s}=0.02 \mathrm{GPa}$ and $\alpha_{f}=\alpha_{m}$ ), while the effects of thermal mismatch are demonstrated in Fig. 4.4 (with $\tau_{s}=0.02 \mathrm{GPa}$ ). Here, and elsewhere in the present work, these effects are calculated for a temperature drop $\Delta T=-550^{\circ} \mathrm{C}$ below a stress-free initial condition at an elevated temperature. Figure 4.5 shows that, for a certain combination of material parameters, it is indeed possible to attain a sign reversal in the lateral strain, as observed in Fig. 1.2. In this figure, $\mathfrak{g}_{m}^{c}=5 \mathrm{~N} / \mathrm{m}$ and $\tau_{s}=0.2 \mathrm{GPa}$.

The radial variations of $\sigma_{z}^{f}$ at the plane of the matrix crack, and its dependence on the residual thermal stress, are shown in Figs. $4.6 \mathrm{a}$ and $4.6 \mathrm{~b}$ for two levels of applied load $\sigma_{a}$ and with $\tau_{s}=0.02 \mathrm{GPa}$. Note that all models based on shear-lag neglect this radial variation and, when matrix cracks are present, cannot account for residual thermal stress effects. The effect of $\tau_{s}$ on $\sigma_{z}^{f}(r / a, \tilde{z} / a)$ is demonstrated in Fig. 4.7 by plotting profiles of these stresses at various distances $\tilde{z} / a$ from the plane of the matrix crack (i.e., $\tilde{z}=z+L / 2$ ) vs $r / a$. Thermal effects are discarded in this figure.

Larger values of $\tau_{s}$ arc associated with larger amplifications and higher peak values of $\sigma_{z}^{f}$ near the plane of the matrix crack, but these amplifications reduce more quickly to the undisturbed background levels upon moving away from the matrix crack. In addition, larger values of $\tau_{s}$ are accompanied by shorter slip zones $l_{s}$ and larger spacings $L$ between matrix cracks. Figures $4.8 \mathrm{a}, 4.8 \mathrm{~b}$, and 4.9 exhibit further details about factors that affect $L$ and $l_{s}$.

Note that Figs. 2.1 and 4.1 represent a highly idealized circumstance of equal fiber spacings $L$ and $L / 2$. It is more reasonable to assume that in realistic circumstances the crack spacings at each stress level $\sigma_{a}$ range between $L_{z}$ and $2 L_{z}$, with an average value of $L=3 / 2 L_{z}$. This suggests that the length $L$ employed in this work should be interpreted to be $50 \%$ larger than the average crack spacing. More refined considerations (Kimber and Keer 1982) suggest that $L=1.337 L_{z}$.

\footnotetext{
"It turned out that at $\sigma_{a}=\sigma_{a}^{c}$, the slip zones near the new cracks in State II (say) had lengths $l_{s}^{\prime}=l_{s}$.

tThis observation explains why the occurrence of early matrix cracks cannot be detected from stress-strain response. In addition, it appears that deterministic analyses that aim at predicting conditions for the onset of first matrix cracking may be futile, because the early state of cracking is entirely governed by stochastic parameters such as the random geometry of initial flaws within the composite.
} 


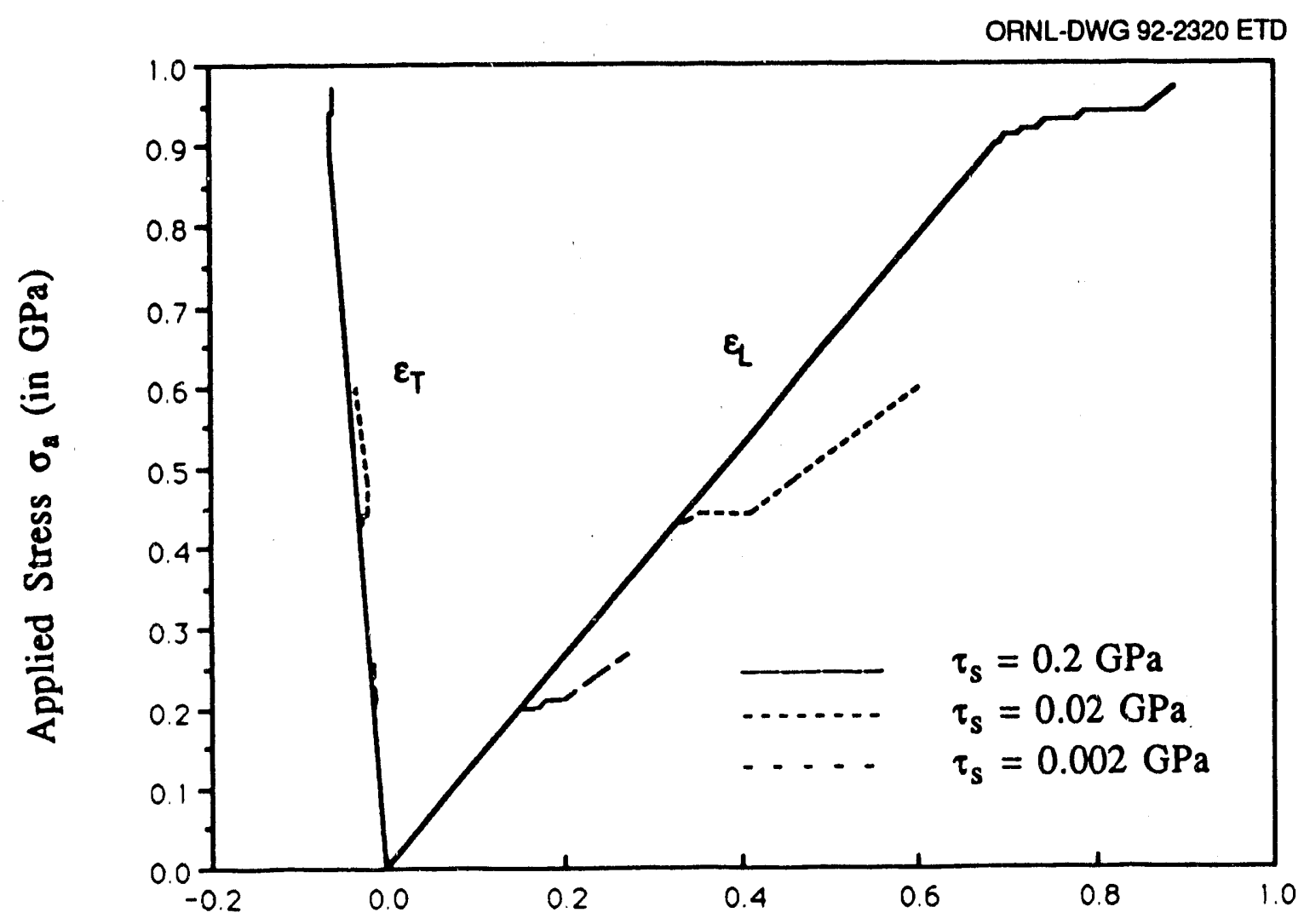

Strain (z)

Fig. 4.2. The effect of interfacial shear strength, $\tau_{s}$, on the stress-strain response of continuous fiber-reinforced ceramic composites (with $\alpha_{f}=\alpha_{m}$ ).

The value of $g_{m}^{c}$ was selected, ad hoc, to be the same as obtained in the monolithic ceramic matrix. In principle, this value is stochastic rather than deterministic. In addition, it is not certain that the in situ value of $\mathfrak{g}_{m}^{c}$ within a composite is in fact the same as for the monolithic material.

The stress-strain plots in Figs. 4.2, 4.3, 4.4, and 4.5 were terminated when $l_{s}=L / 2$, namely when slip occurred over the entire fiber/matrix interface. It seems that beyond that range the stress-strain behavior is governed by fiber failures. 


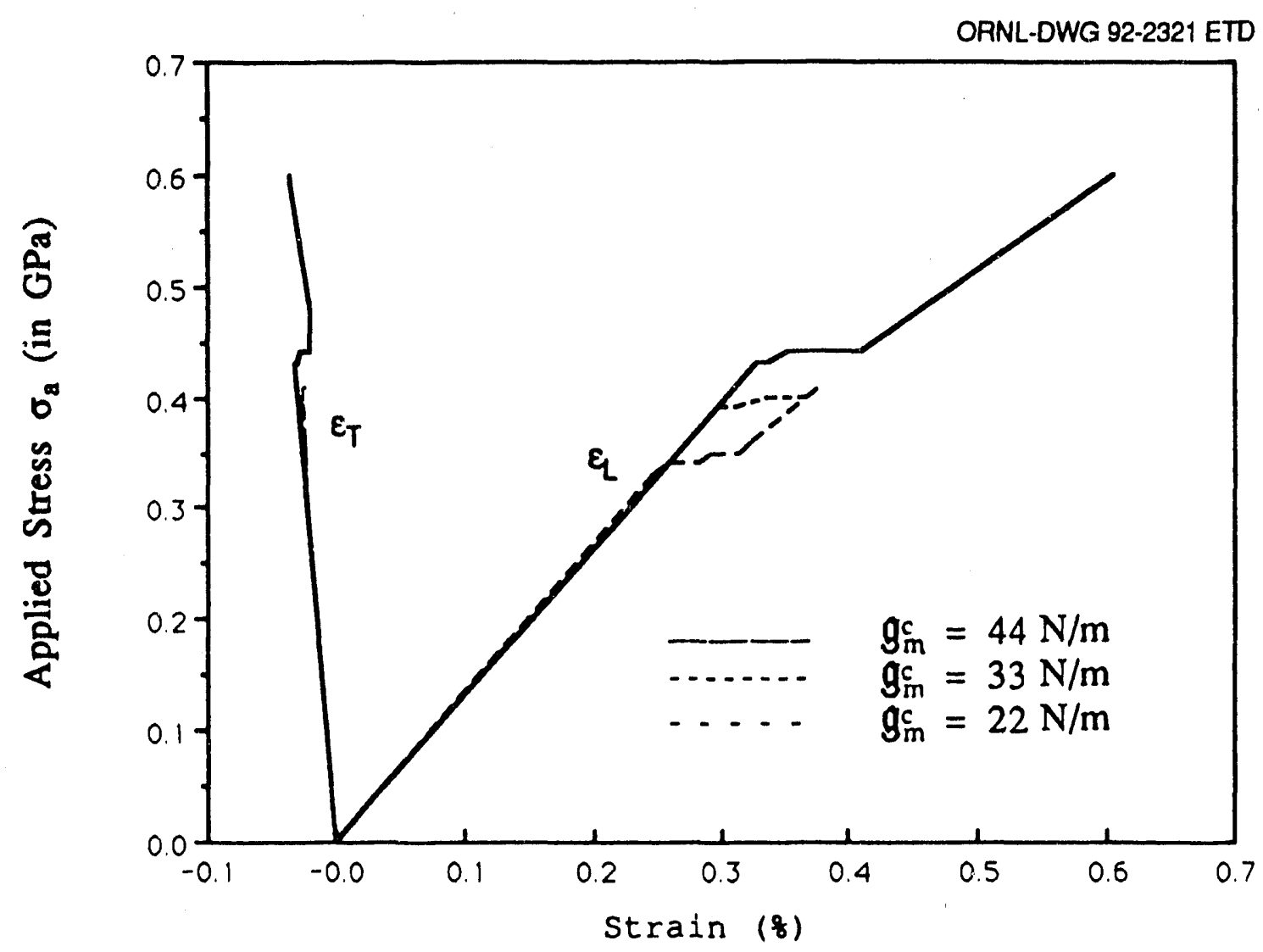

Fig. 4.3. Effects of matrix fracture toughness on stress-strain response of continuous fiber-reinforced ceramic composites $\left(\tau_{s}=0.02, \alpha_{f}=\alpha_{m}\right)$. 


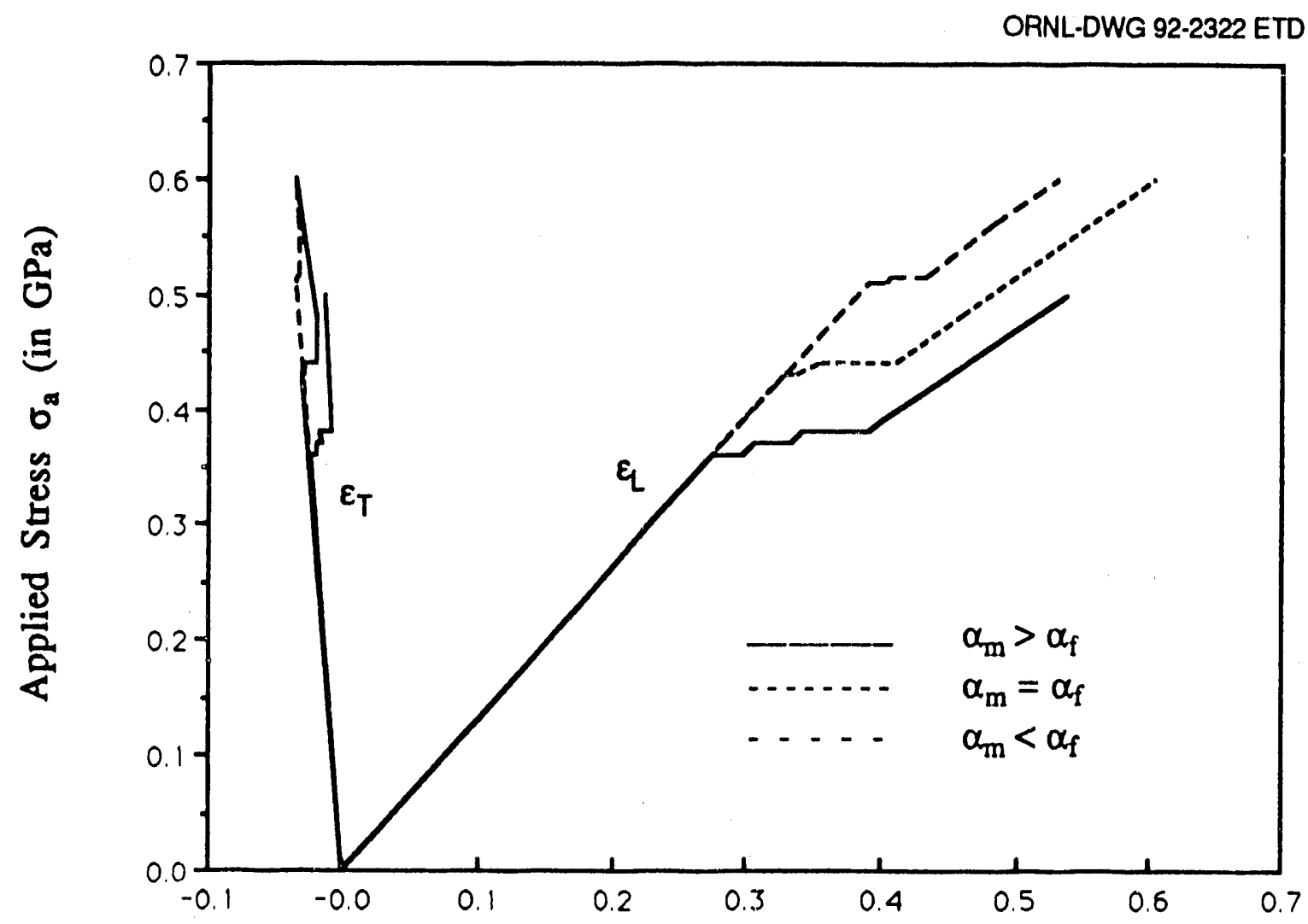

Strain (8)

Fig. 4.4. Effect of thermal expansions on stress-strain response of continuous fiberreinforced ceramic composites $\left(\boldsymbol{\tau}_{\boldsymbol{s}}=\mathbf{0 . 0 2}\right)$. 


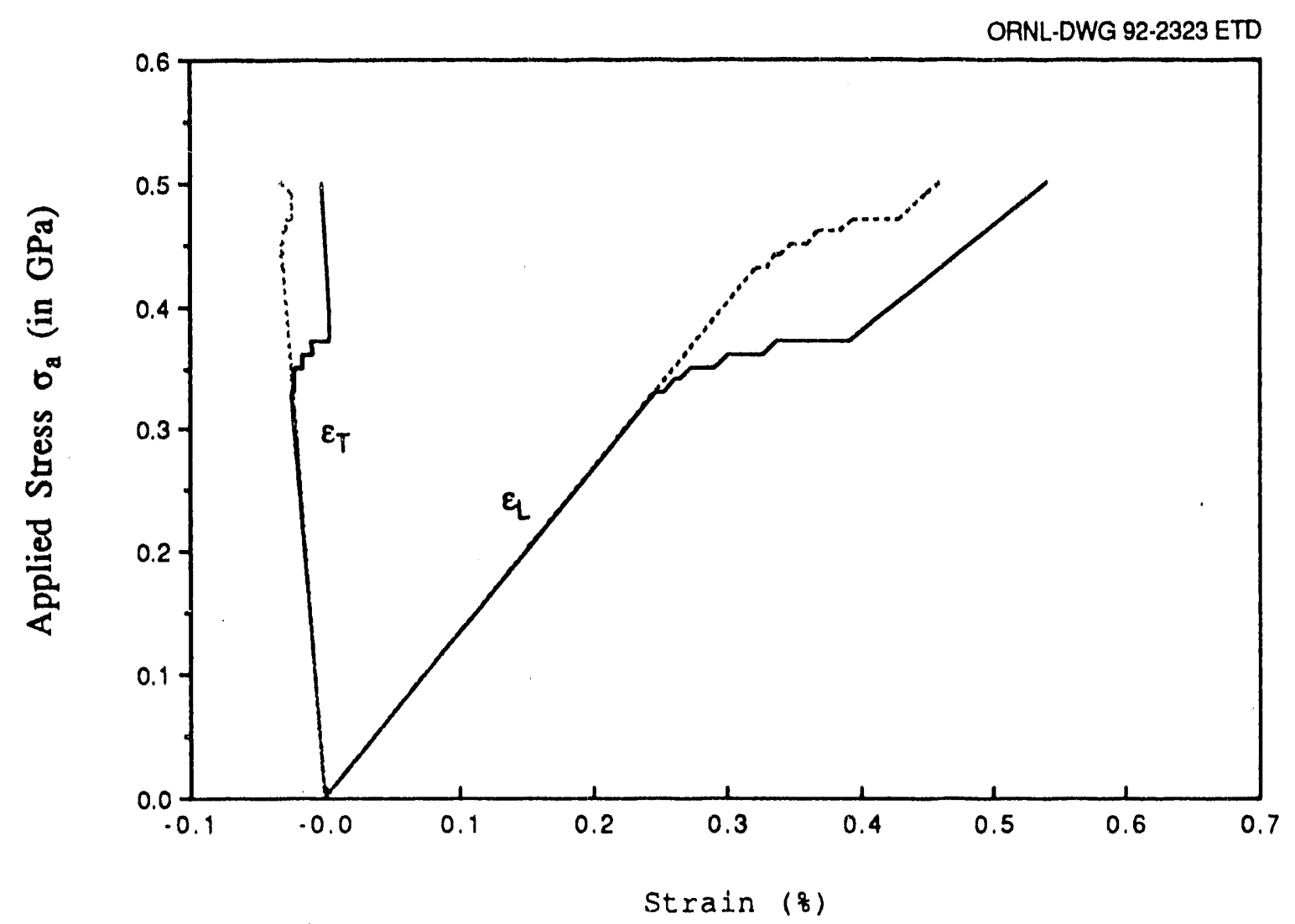

Fig. 4.5. Stress-strain response at $G_{m}^{c}=5 \mathrm{~N} / \mathrm{m}$ and $\tau_{s}=0.2 \mathrm{GPa}$ for $\alpha_{m}>\alpha_{f}(\longrightarrow)$ and $\alpha_{m}=\alpha_{f}(-\cdots)$. 


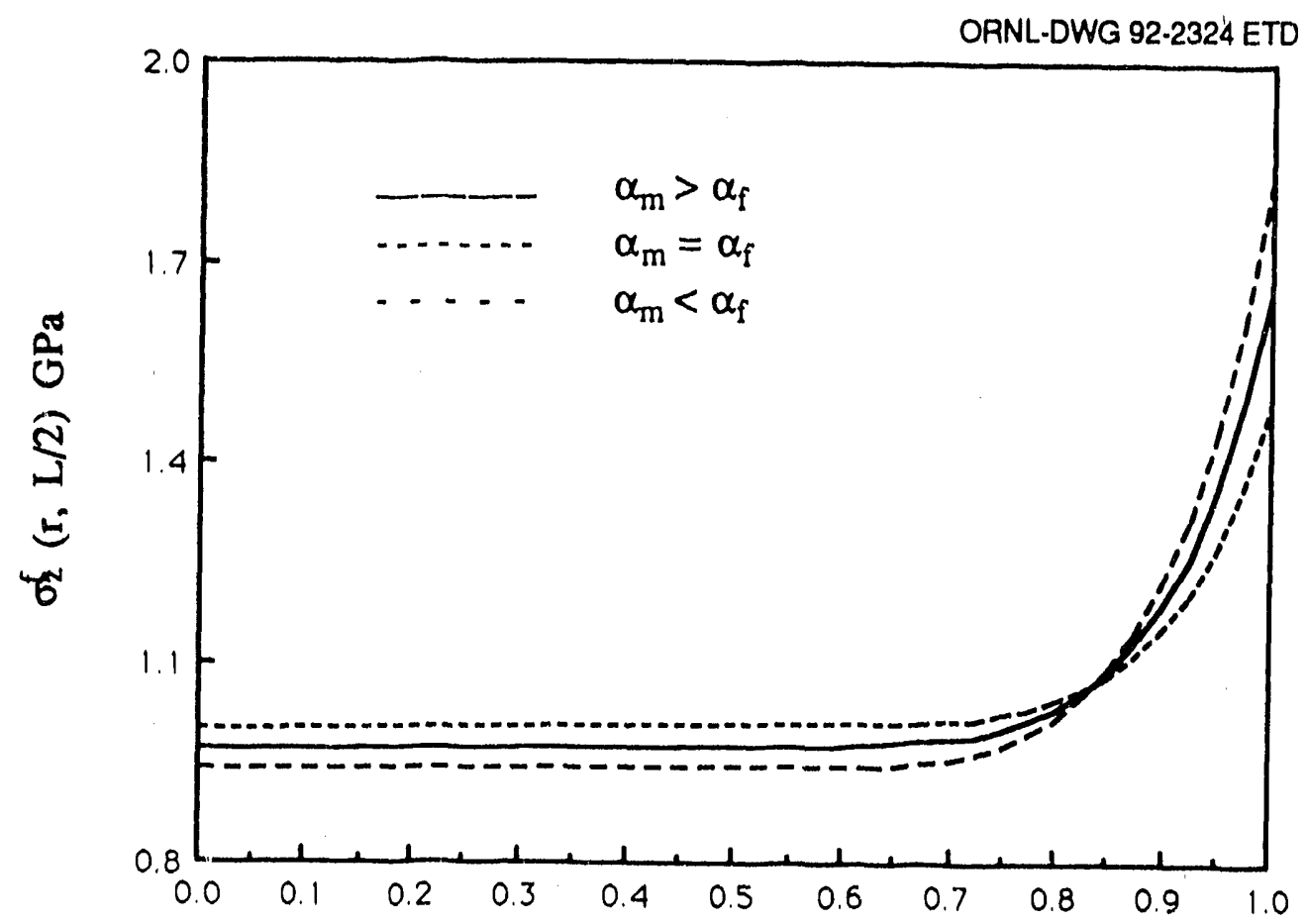

(a)

$r / a$

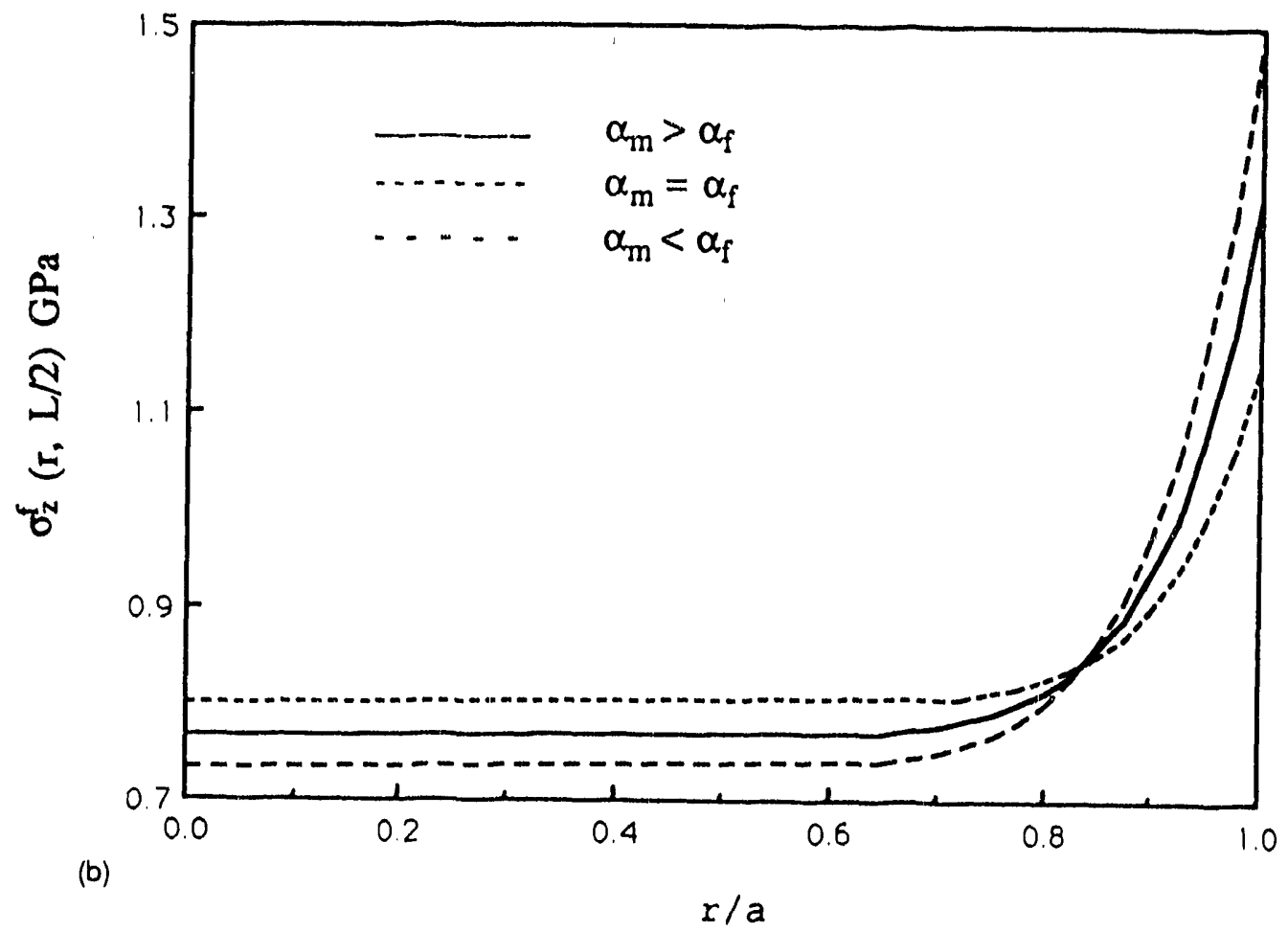

Fig. 4.6. Effect of thermal expansions on stress distribution within fiber (at plane of matrix cracks) (a) $\sigma_{a}=0.43 \mathrm{GPa}\left(\tau_{s}=0.02 \mathrm{GPa}\right),(\mathrm{b}) \sigma_{a}=0.34 \mathrm{GPa}\left(\tau_{s}=0.02 \mathrm{GPa}\right)$. 

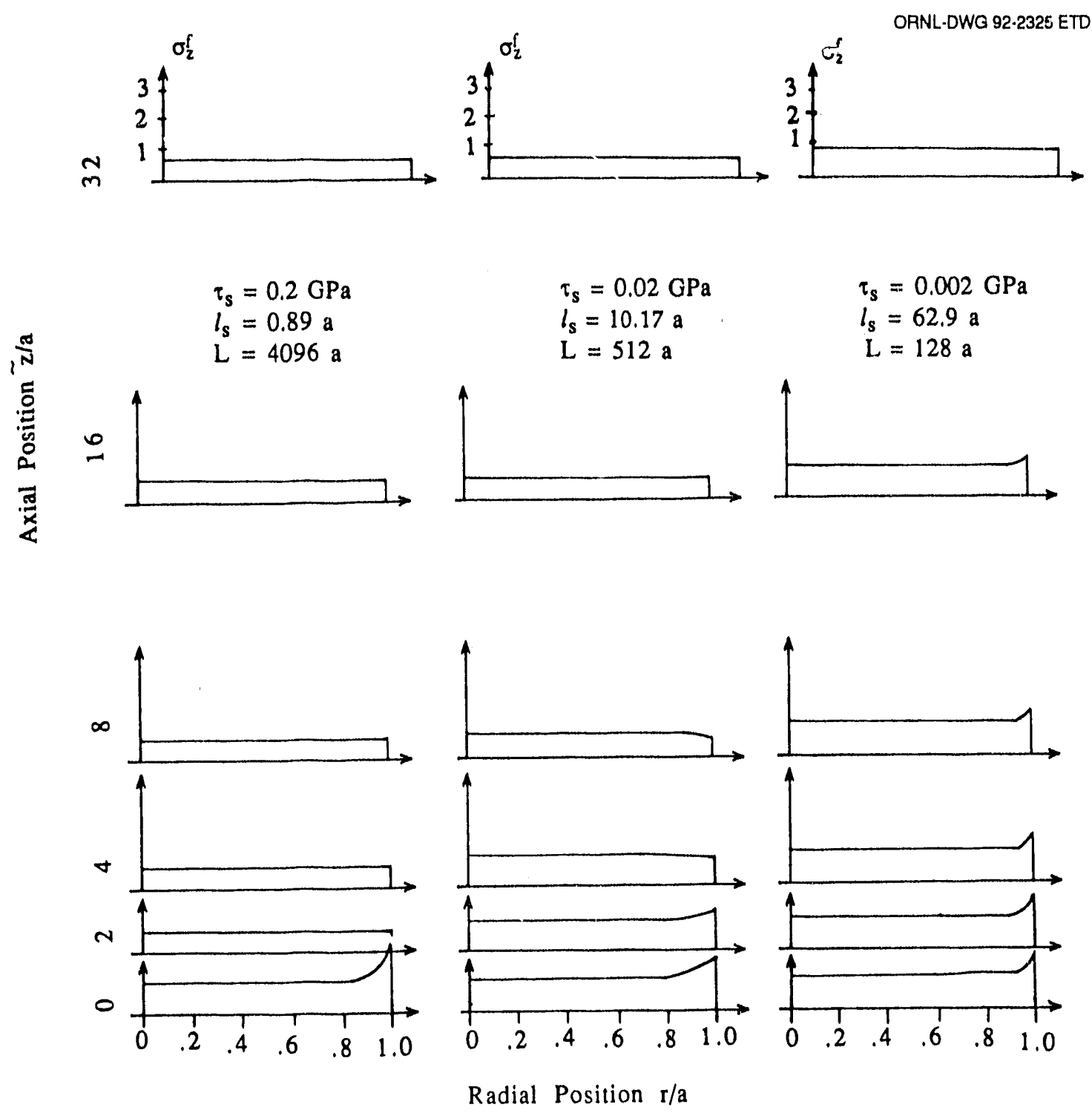

Fig. 4.7. Dependence of stress distributions within fibers on the interfacial shear strength $\tau_{s}$. Shown are profiles of $\sigma_{z}^{f}(r / a, \tilde{z} / a)$ (in GPa) at various distances, $\tilde{z} / a$, from the matrix crack, for $\sigma_{a}=0.43 \mathrm{GPa}$. 

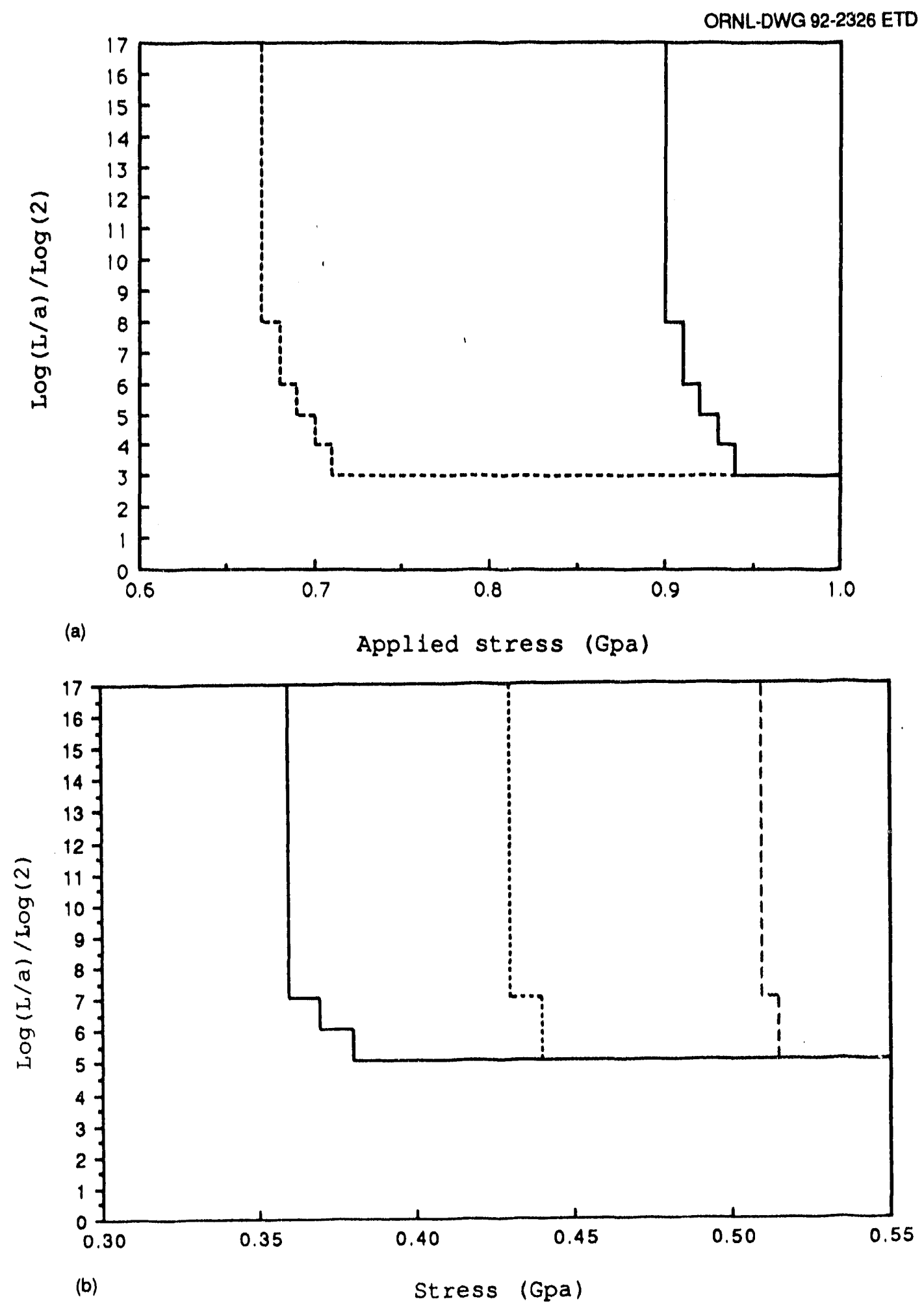

Fig. 4.8. Crack spacing, $L$, in terms of $\log (L / a) / \log (2)$ vs applied stress for (a) $\tau_{s}=$ $0.2 \mathrm{GPa}$. Cases of $\alpha_{m}=\alpha_{f}$ (solid line) and $\alpha_{m}>\alpha_{f}$ (dashed line). (b) $\tau_{s}=0.02 \mathrm{GPa}$. Cases of $\alpha_{m}>\alpha_{f}(-), \alpha_{m}=\alpha_{f}(\ldots-\ldots)$, and $\alpha_{m}<\alpha_{f}(\ldots \ldots)$. 


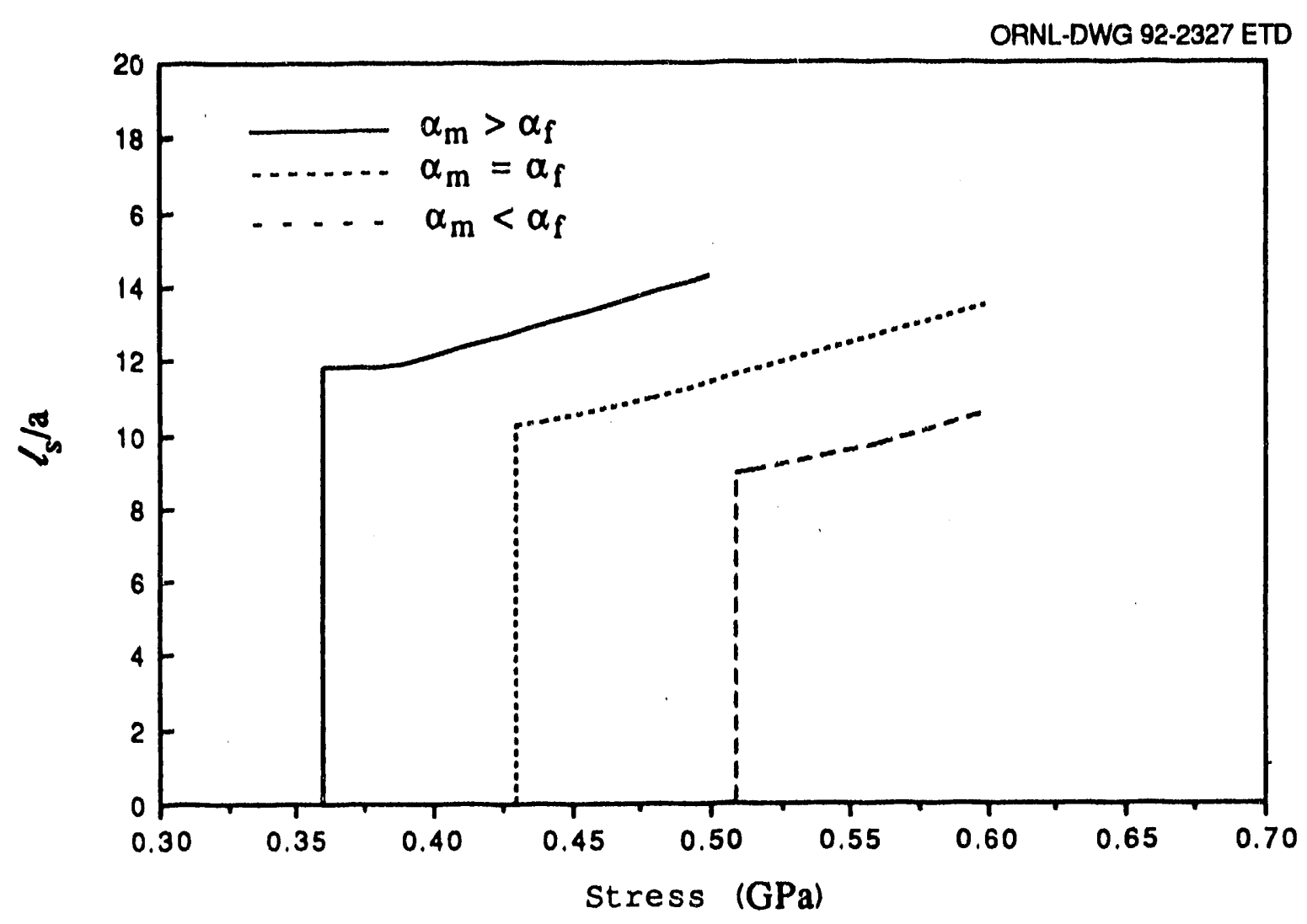

Fig. 4.9. Length of slip zone, $l_{s}$, vs applied stress for $\tau_{s}=0.02 \mathrm{GPa}$. Cases of $\alpha_{m}>\alpha_{f}$ $\left(\longrightarrow, \alpha_{m}=\alpha_{f}(\cdots)\right.$, and $\alpha_{m}<\alpha_{f}(\cdots)$. 


\section{STATISTICAL PREDICTIONS OF FIBER FAILURES}

It is generally agreed that fiber fallure is controlled by a statistical distribution of microstructural surface flaws (Thouless and Evans 1988, Sutcu 1989). The effect of these flaws on the statistics of fiber strength is commonly represented by a Weibull distribution, which is also adopted in the present case. Accordingly, the probability $P_{f}$ of fiber failure is expressed by

$$
P_{f}=1-\exp \left\{-2 \pi a N \int_{-L / 2}^{L / 2}\left[\frac{\sigma_{z}^{f}(a, z)}{\sigma_{0}}\right]^{M} d z\right\} \text {, }
$$

where $2 \pi a L$ is the outer cylindrical surface of the fiber within an RVE of length $L ; N=L^{*} / L$ is the number of matrix cracks in a test coupon of length $L^{*} ; M$ is the Weibull modulus that, for ceramic fibers, has values between 8 and 10; and $\sigma_{0}$ is a Weibull parameter that represents, approximately, the mean value of the failure stress.

Note also that, because $M>1$, we have at any cross section $A_{f}=\pi a^{2}$ within the RVE $\int_{A}\left[\sigma_{z}^{f}(r ; z)-\bar{\sigma}_{z}^{f}(z)\right]^{M} d A_{f}>0$, where $\bar{\sigma}_{z}^{f}(z)$ is the average stress at any $z$. It follows that fiber failure predictions based on models that do not detail the radial variation of $\bar{\sigma}_{z}^{f}$ are likely to grossly underestimate the failure probability. This occurs for the commonly employed shear-lag model.

The probabilities $P_{f}$ of fiber failure under increasing levels of applied stress $\sigma_{a}$ were computed in accordance with Eq. (45), employing values for $\sigma_{z}^{f}(a, z)$ and $L$ determined by the formulations in Sects. 3 and 4. We also employed the values $M=10$ and $\sigma_{0}=0.4 \mathrm{GPa} \mathrm{m}^{2 / M}$.

Some aspects of the dependence of $P_{f}$ on the interfacial shear strength $\tau_{s}$ are exhibited in Fig. 5.1, where $P_{f}$ is plotted vs $\sigma_{a}$ for $\tau_{s}=0.2,0.02$, and $0.002 \mathrm{GPa}$. In these computations, it was also assumed that $\alpha_{f}=\alpha_{m}$. The effect of residual thermal stresses, with $\tau_{s}=0.02 \mathrm{GPa}$, is shown in Fig. 5.2, while the significant effect of the radial variation of $\sigma_{z}^{f}$ on $P_{f}$ is demonstrated in Figs. 5.3a and 5.3b. Note that computations based on radially averaged values $\bar{\sigma}_{z}^{f}(z)$ will underpredict the probabilities of fiber failure.

As noted by Schwietert and Steif (1990), multiple failures may occur within a single fiber. Such failures and, especially, denser crack spacing as $P_{f} \rightarrow 1$ would necessitate the consideration of fiber failure interactions. Fiber failures will modify the stress-strain curves shown in Figs. 4.2 through 4.5 and, most importantly, will introduce another energy-absorbing mechanism, commonly referred to as "fiber pull-out," that adds significantly to the toughening of the composite. 


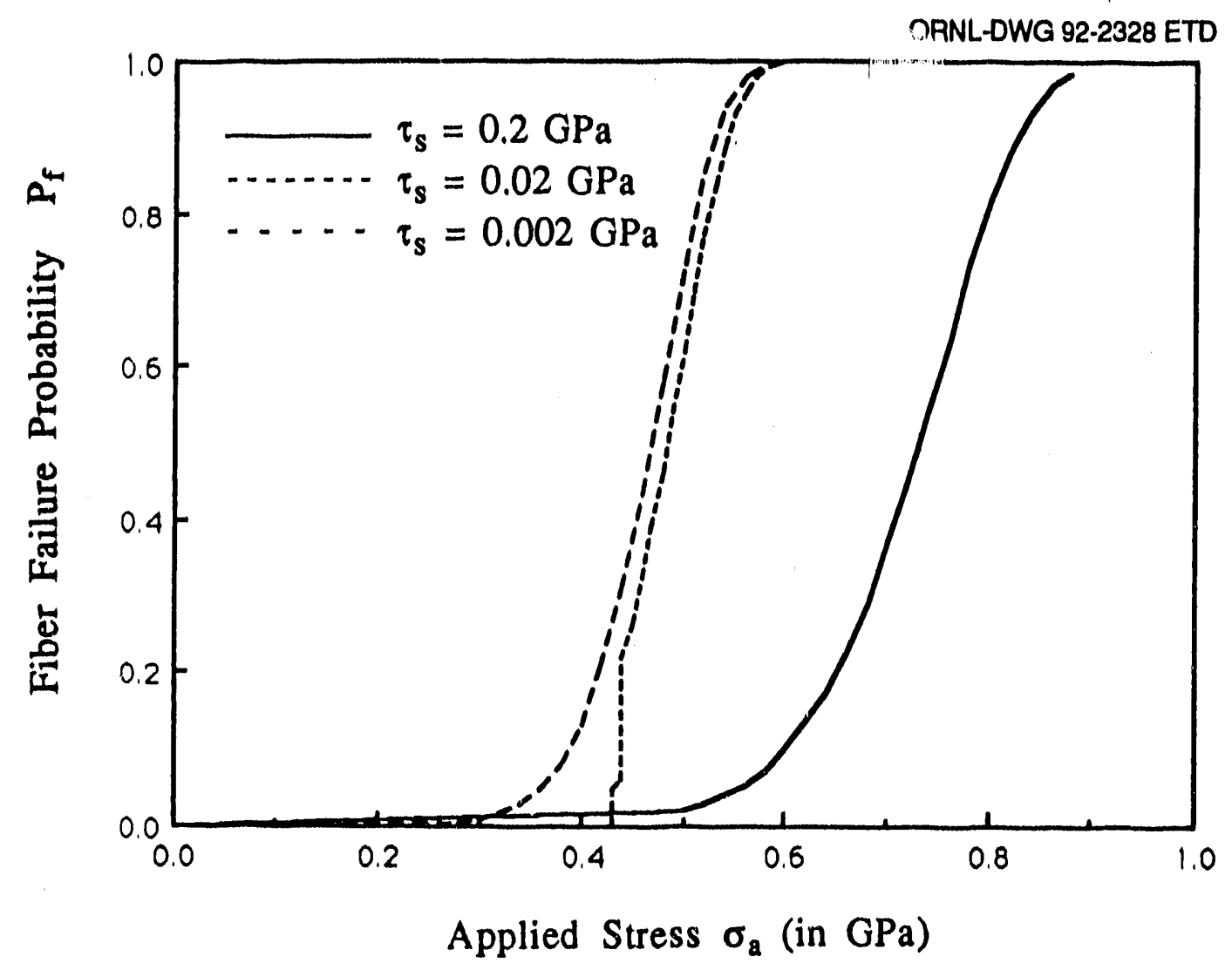

Fig. 5.1. The effect of interfacial shear stress, $\tau_{s}$, (in $\mathbf{G P a}$ ) on the probability of fiber failure, accounting for radial variation in fiber stress distribution (with $\alpha_{f}=\alpha_{m}$ ). 


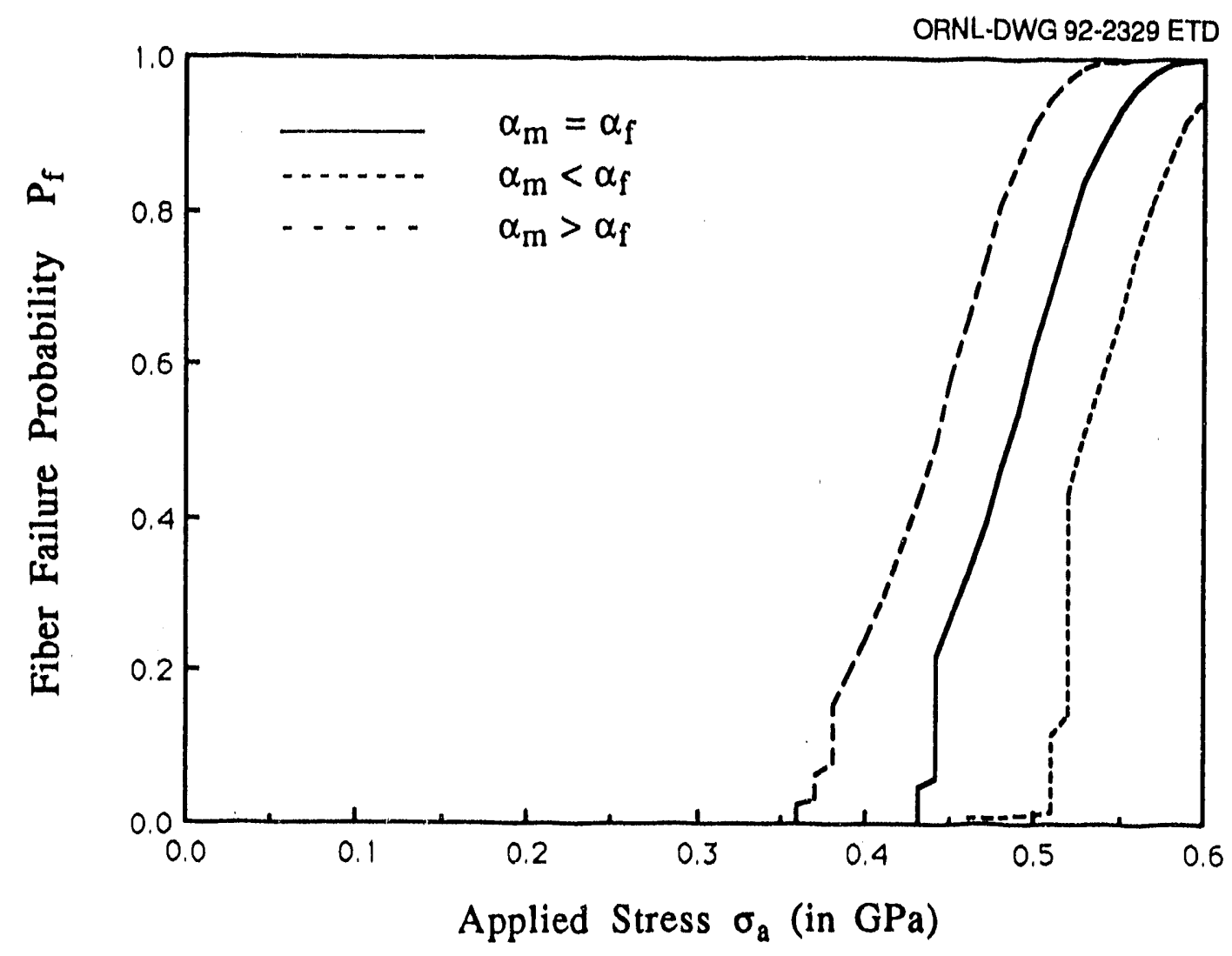

Fig. 5.2. Probability of fiber failure vs applied stress and its dependence on thermal expansions $\left(\tau_{s}=0.02 \mathrm{GPa}\right)$. 


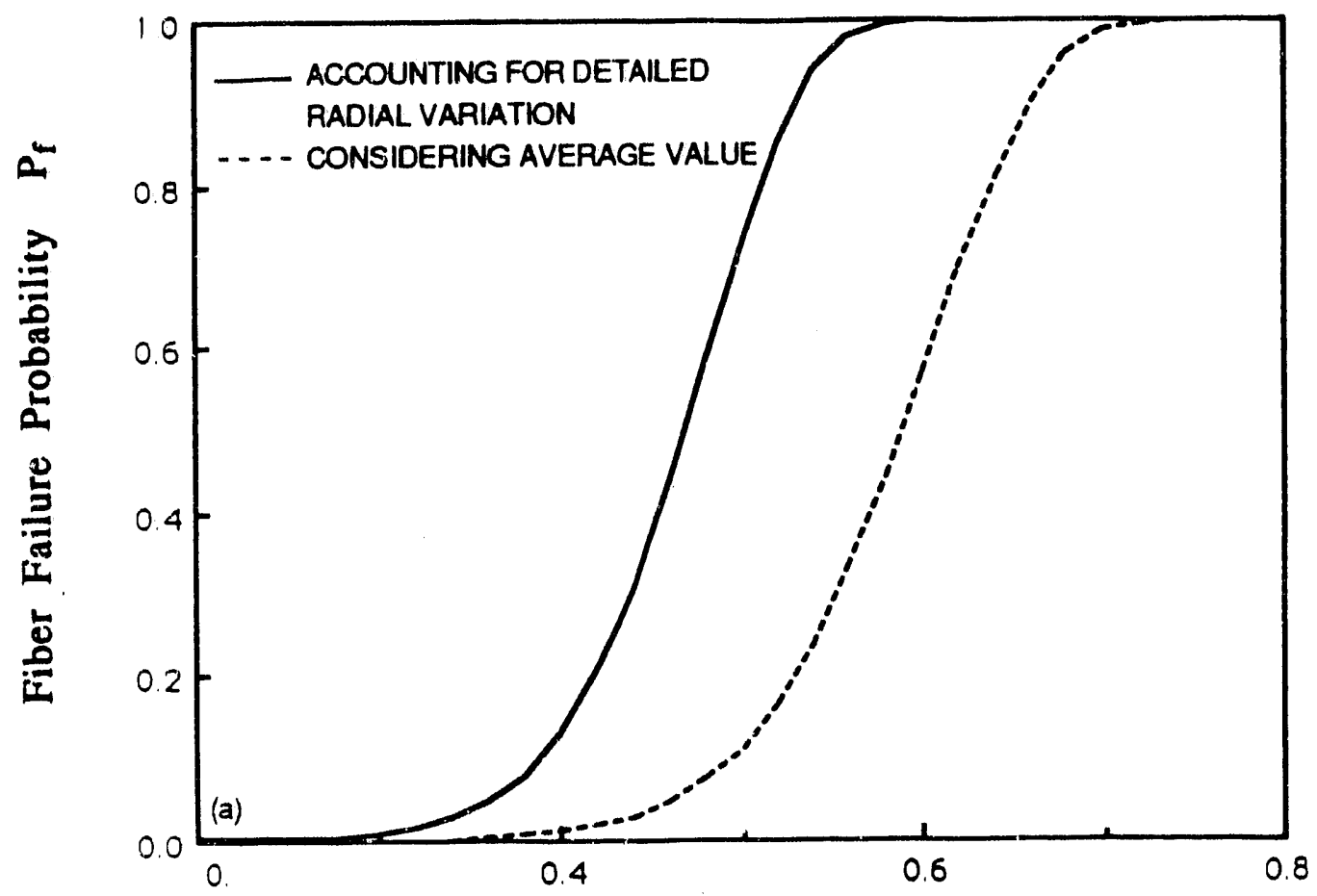

Applied Stress $\sigma_{a}$ (in GPa)

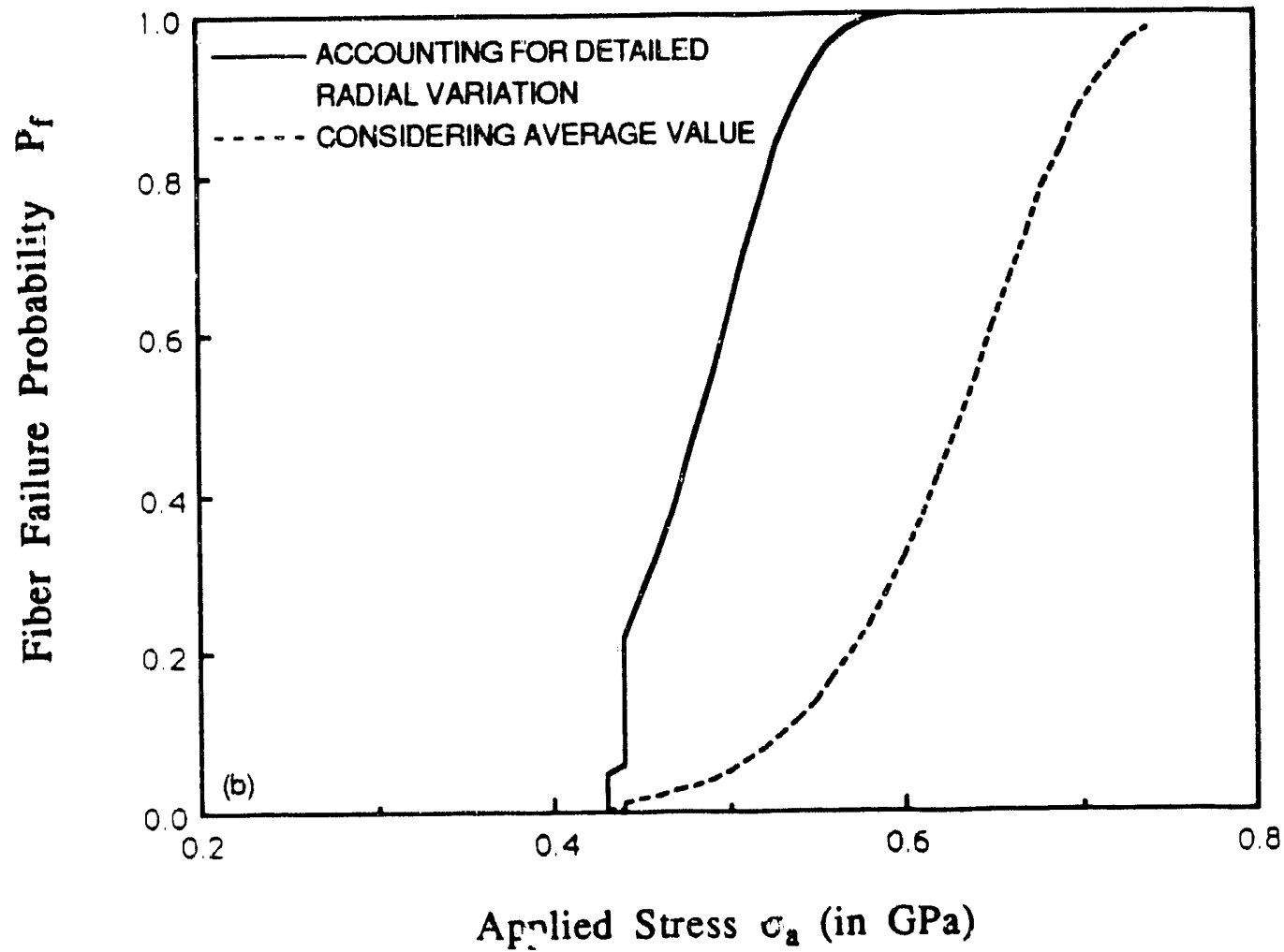

Fig. 5.3. The effect of radial variation of fiber stresses on the probability of fiber failure (a) $\left(\tau_{s}=0.002 \mathrm{GPa}, \alpha_{m}=\alpha_{f}\right)$, (b) $\left(\tau_{s}=0.02 \mathrm{GPa}, \alpha_{m}=\alpha_{f}\right)$. 


\section{SOME CONSIDERATIONS OF FIBER PULL-OUT ENERGY}

It is now hypothesized that a negligible amount of energy is dissipated when a fiber break occurs within the perfectly bonded contact region of an RVE, namely, at location $z$ equal or equivalent to $0<z<L / 2-l_{s}$. In these circumstances, the load bome by the fiber is transferred to the matrix, and the effect of the fiber break is localized within just a few fiber diameters (Kelly and MacMillan 1986). In addition, because the site of such a fiber crack is somewhat far from the location of any matrix crack, interactive failure is less likely to occur.

On the other hand, when fiber failure occurs within the slip zone, that is, at a location $z_{f b}$, where $L / 2-l_{s}<z_{f b}<L / 2$, the effect of the fiber break extends all the way to $z=L / 2$. The reason for this longer range effect is demonstrated in Fig. 6.1.

Accordingly, at $z_{f b}<z<L / 2, r=a$, the shear stresses $\tau_{f b}$ due to a fiber break at $z=z_{f b}$ would point in the same direction as the shear stresses $\tau_{s}$ caused by the pre-existing matrix crack at $z=$

- $L / 2$, while for $z>L / 2$ and $z<z_{f b}, \tau_{f b}$ and $\tau_{s}$ point in opposite directions. Hiowever, because the interfacial shear stresses cannot exceed $\tau_{s}$, the fiber region $z_{f b}<z<L / 2,0 \leq r<a$, will contract without frictional resistance at $r=a$, namely as a "free-standing column."

Because fiber failure in the slip zones is a random event, we now evaluate the probability $P_{f s}$ of such occurrence. This quantity is to be contrasted with $P_{f}$, the probability of fiber failure anywhere along its length, given in Eq. (45).

For that purpose, we introduce the function

$$
P_{f}(0, z)=1-\exp \left\{-2 \pi a \int_{0}^{z}\left[\frac{\sigma_{z}^{f}(a, \hat{z})}{\sigma_{0}}\right]^{M} d \hat{z}\right\}
$$

which expresses the probability of fiber failure within the interval $(0, z)$.

Accordingly, the probability of failure within the interval $z_{1}<z<z_{2}$ is given by

$$
\begin{aligned}
P_{f}\left(z_{1}, z_{2}\right) & =\int_{z_{1}}^{z_{2}} \frac{\partial P_{f}(0, z)}{\partial z} d z \\
& =\exp \left\{-2 \pi a \int_{0}^{z_{1}}\left[\frac{\sigma_{z}^{f}(a, z)}{\sigma_{0}}\right]^{M} d z\right\}-\exp \left\{-2 \pi a \int_{0}^{z_{2}}\left[\frac{\sigma_{z}^{f}(a, z)}{\sigma_{0}}\right]^{M} d z\right\} \\
& \left.=\exp \left\{-2 \pi a \int_{0}^{z_{1}}\left[\frac{\sigma_{z}^{f}(a, z)}{\sigma_{0}}\right]^{M} d z\right\} 1-\exp \left\{-2 \pi a \int_{z_{1}}^{z_{2}}\left[\frac{\sigma_{z}^{f}(a, z)}{\sigma_{0}}\right]^{M} d z\right\}\right)
\end{aligned}
$$

It can be readily verified that $P_{f}\left(z_{1}, z_{2}\right)$ possesses the required property that, for any $z_{1}<z_{2}<z_{3}$,

$$
P_{f}\left(z_{1}, z_{3}\right)=P_{f}\left(z_{1}, z_{2}\right)+P_{f}\left(z_{2}, z_{3}\right) \text {, }
$$

which is consistent with the Weibull statistical approach employed in the present framework 
State "0": No Cracks

ORNL-DWG 92-2331 ETD

$\sigma_{a} \leftarrow\left\{\begin{array}{cc}\text { m } & \sigma_{m}^{(0)} \\ \hline \text { ( ) } & \sigma_{p}^{(0)} \\ \text { m } & \sigma_{m}^{(0)}\end{array} \rightarrow \sigma_{a}\right.$

State "1": A Matrix Crack at $z=L / 2$ with Interfacial Slip Zones

$$
-\sigma_{m}^{(0)}
$$
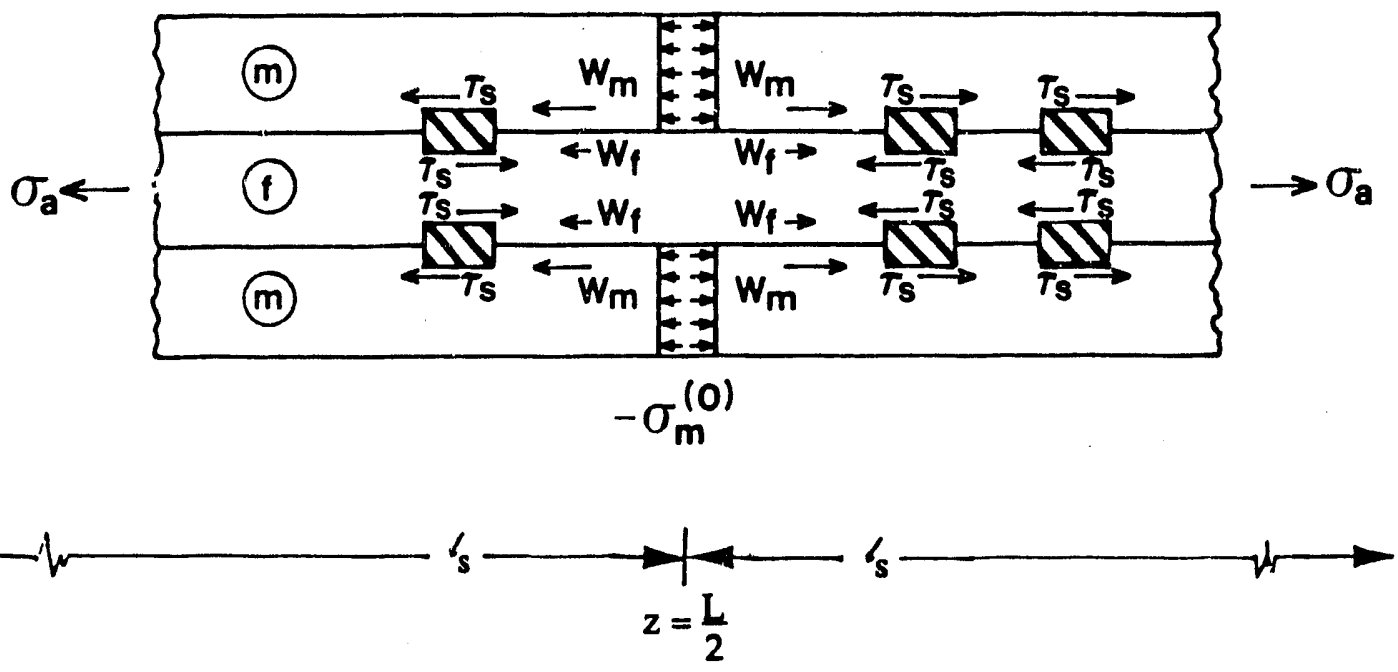

State "2": Fiber Break at $z=z_{f b}$ Superimposed on Matrix Crack at $z=L / 2$

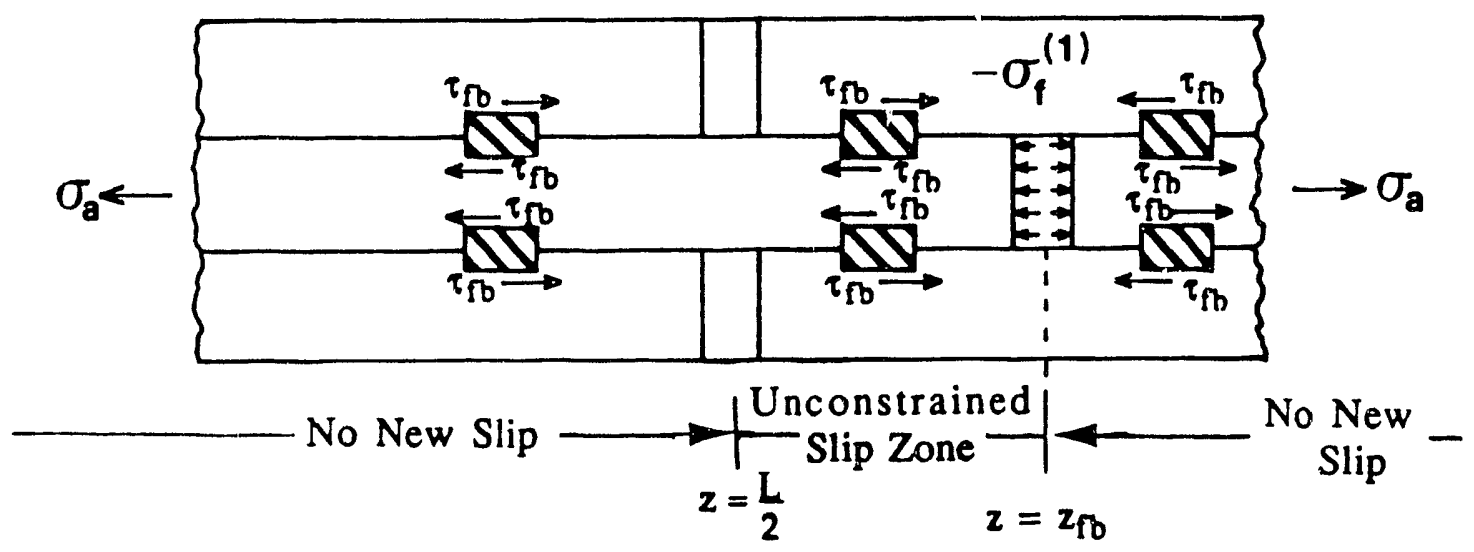

Fig. 6.1. A sketch of the pull-out mechanism when a matrix crack at $z=L / 2$ is followed by a fiber break at $z=z_{f b}$. 
Employing Eqs. (47) and (48), together with mathematical induction, yields the following expression for the probability of failure within the $2 N$ slip zones, each of length $l_{s}$, that exist along the specimen of length $N L$ :

$$
P_{f s}=P_{f} \exp \left\{-2 \pi a \int_{0}^{L / 2-l_{s}}\left[\frac{\sigma_{z}(a, z)}{\sigma_{0}}\right]^{M} d z\right\} \times \frac{1-\exp \left\{-4 \pi a \int_{L / 2-l_{s}}^{L / 2}\left[\frac{\sigma_{z}(a, z)}{\sigma_{0}}\right]^{M} d z\right\}}{1-\exp \left\{-4 \pi a \int_{0}^{L / 2}\left[\frac{\sigma_{z}(a, z)}{\sigma_{0}}\right]^{M} d z\right\}},
$$

with $P_{f}$ given in Eq. (45).

In the derivation of expression (49), we utilized the periodicity of the $\sigma_{z}^{f}$, namely, $\sigma_{z}^{f}(a, z$ $+k L)=\sigma_{z}^{f}(a, z)(k= \pm 1, \pm 2, \ldots)$. For $\tau_{s}=0.02 \mathrm{GPa}$ and $\tau_{s}=0.002 \mathrm{GPa}, P_{f s}$ is indistinguishable from $P_{f}$, hence $P_{f s}$ vs $\sigma_{a}$ are the same as shown in Fig. 5.1.

For $\tau_{s}=0.2 \mathrm{GPa}, P_{f s}$ differs from $P_{f}$. These failure probabilities are shown vs $\sigma_{a}$ in Fig. 6.2. Inspection of Figs. 4.2, 5.1, and 6.2 suggests that for $\tau_{s}=0.02 \mathrm{GPa}$ and $\tau_{s}=0.002 \mathrm{GPa}$, the

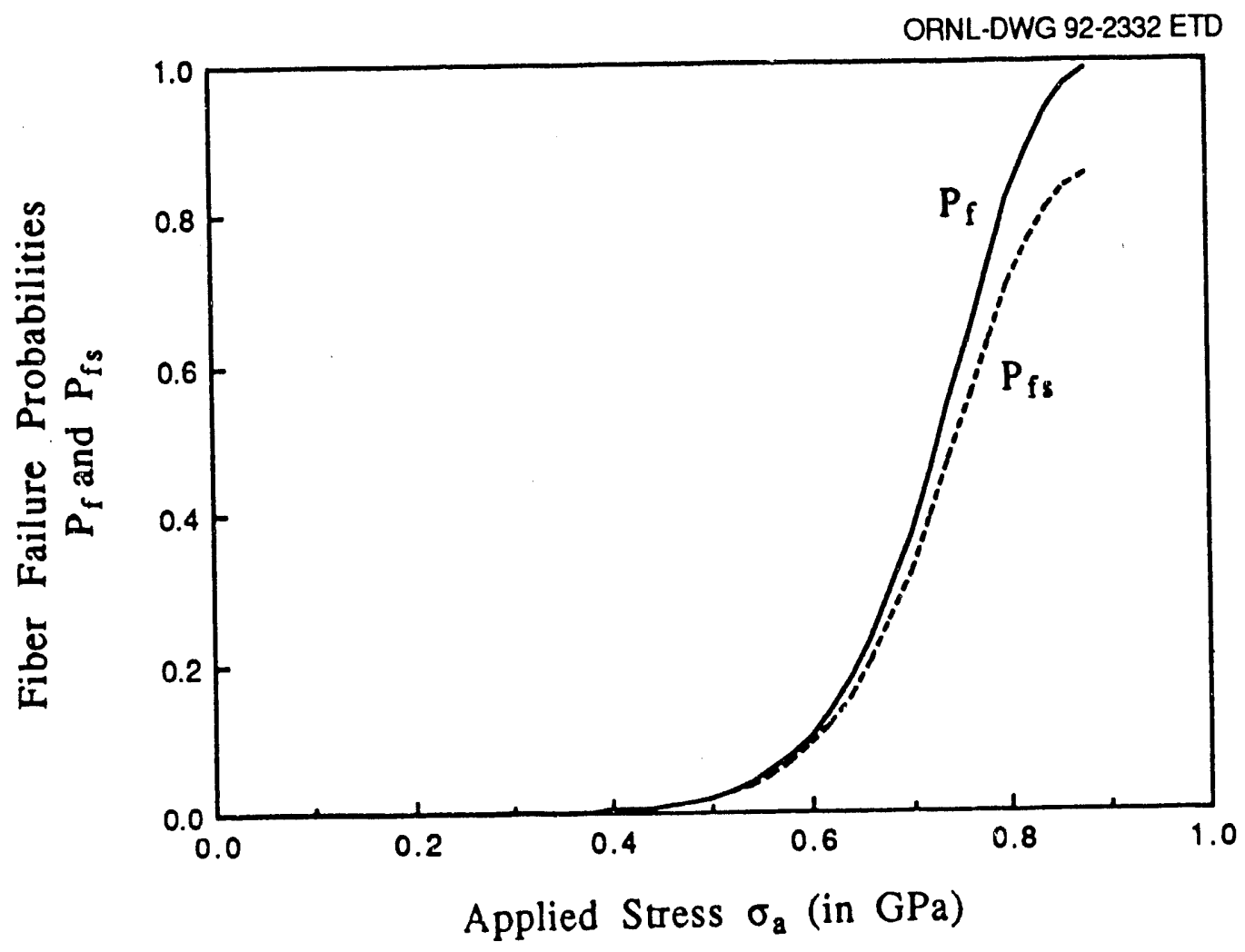

Fig. 6.2. A comparison between $\boldsymbol{P}_{f}$ the probability of fiber failure (solid line), and $\boldsymbol{P}_{f s}$, the probability of fiber failure within a slip zone (dashed line), vs the applied stress $\sigma_{a}$. Case of $\tau_{s}=0.2 \mathrm{GPa}$ and $\alpha_{m}=\alpha_{f}$ 
incidence of matrix cracking antedates fiber failures, confirming the scenario illustrated in Fig. 6.1. However, for the case of strong interface, that is, $\tau_{s}=0.2 \mathrm{GPa}$, fiber failures precede matrix cracking.

Employing Eq. (47), we obtain the probability of fiber failure within a single slip zone of a single RVE:

$P_{f}\left(\frac{L}{2}-l_{s}, \frac{L}{2}\right)=\exp \left\{-2 \pi a \int_{0}^{L / 2-l_{s}}\left[\frac{\sigma_{z}^{f}(a, z)}{\sigma_{0}}\right]^{M} d z\right\}\left(1-\exp \left\{-2 \pi a \int_{L / 2-l_{s}}^{L / 2}\left[\frac{\sigma_{z}^{f}(a, z)}{\sigma_{0}}\right]^{M} d z\right\}\right)$

The most likely location $z_{f b}^{*}$ of a fiber break within the above slip zones can be evaluated as follows:

$$
z_{f b}^{*} \int_{0}^{\sigma_{a}} \int_{L / 2-l_{s}}^{L / 2} \frac{\partial^{2} P_{f}\left(L / 2-l_{s}, z\right)}{\partial z \partial \sigma_{a}} d z d \sigma_{a}=\int_{0}^{\sigma_{a}} \int_{L / 2-l_{s}}^{L / 2} \frac{\partial^{2} P_{f}\left(L / 2-l_{s}, z\right)}{\partial z \partial \sigma_{a}} z d z d \sigma_{a}
$$

Integration by parts yields

$$
z_{f b}^{*} P_{f}\left(\frac{L}{2}-l_{s}, \frac{L}{2}\right)=P_{f}\left(\frac{L}{2}-l_{s}, \frac{L}{2}\right) \frac{L}{2}-\int_{L / 2-l_{s}}^{L / 2} P_{f}\left(\frac{L}{2}-l_{s}, z\right) d z
$$

Upon determining $z_{f b}^{*}$, it is possible to evaluate $U_{p}$, the portion of the pull-out energy resulting from the unresisted contraction of the fiber in the region $z_{f b}^{*}<z<L / 2$. We have

$$
U_{p}=\frac{P_{f}\left(L / 2-l_{s}, L / 2\right)}{2 E_{f}} \int_{L / 2-z_{f b}^{*}}^{L / 2} \int_{0}^{a}\left\{\left[\sigma_{z}^{f}(r, z)\right]^{2}-\left[\sigma_{z}^{f}(r, z)-\sigma_{z}^{f}\left(r, z_{f b}^{*}\right)\right]^{2}\right\} r d r d z
$$

In the evaluation of $U_{p}$, we accounted only for the release of $\sigma_{z}^{f}$ due to the creation of a fiber crack at $z=z_{f b}^{*}$. The cintributions to all other stress components have been discarded in view of their relative insignificance.

The nondimensionalized values of $U_{p} / \pi\left(b^{2}-a^{2}\right) g_{m}^{c}$ vs $\sigma_{a}$ are plotted in Fig. 6.3 for $\tau_{s}=$ $0.002,0.02$, and $0.2 \mathrm{GPa}$. Note the vast disparities between the magnitudes of $U_{p}$ for these distinct values of $\tau_{s}$. (The plots for $U_{p}$ were terminated at $P_{f}=1$.)

It is important to note that when a matrix crack at $z=L / 2$ is accompanied by a fiber break at $z_{f b}^{*}$ within the slip zone, the RVE can no longer support the remote load $\pi b^{2} \sigma_{a}$. The unbalanced load of magnitude

$$
\pi b^{2} \sigma_{a}-P_{f}\left(\frac{L}{2}-l_{s}, \frac{L}{2}\right) \int_{0}^{a} \sigma_{z}^{f}\left(r, z_{f b}^{*}\right) r d r
$$

is transferred to the neighboring RVEs by shear at $r=b$. The above unbalanced load will overburden the neighboring RVEs and may bring about their premature failure. This process may explain the observed clustering of failures in the form of fiber pull-outs. However, the local failure of the RVE, accompanied by the overloading of its neighboring elements, leads us beyond the scope of the present model and requires a separate analytical approach. 


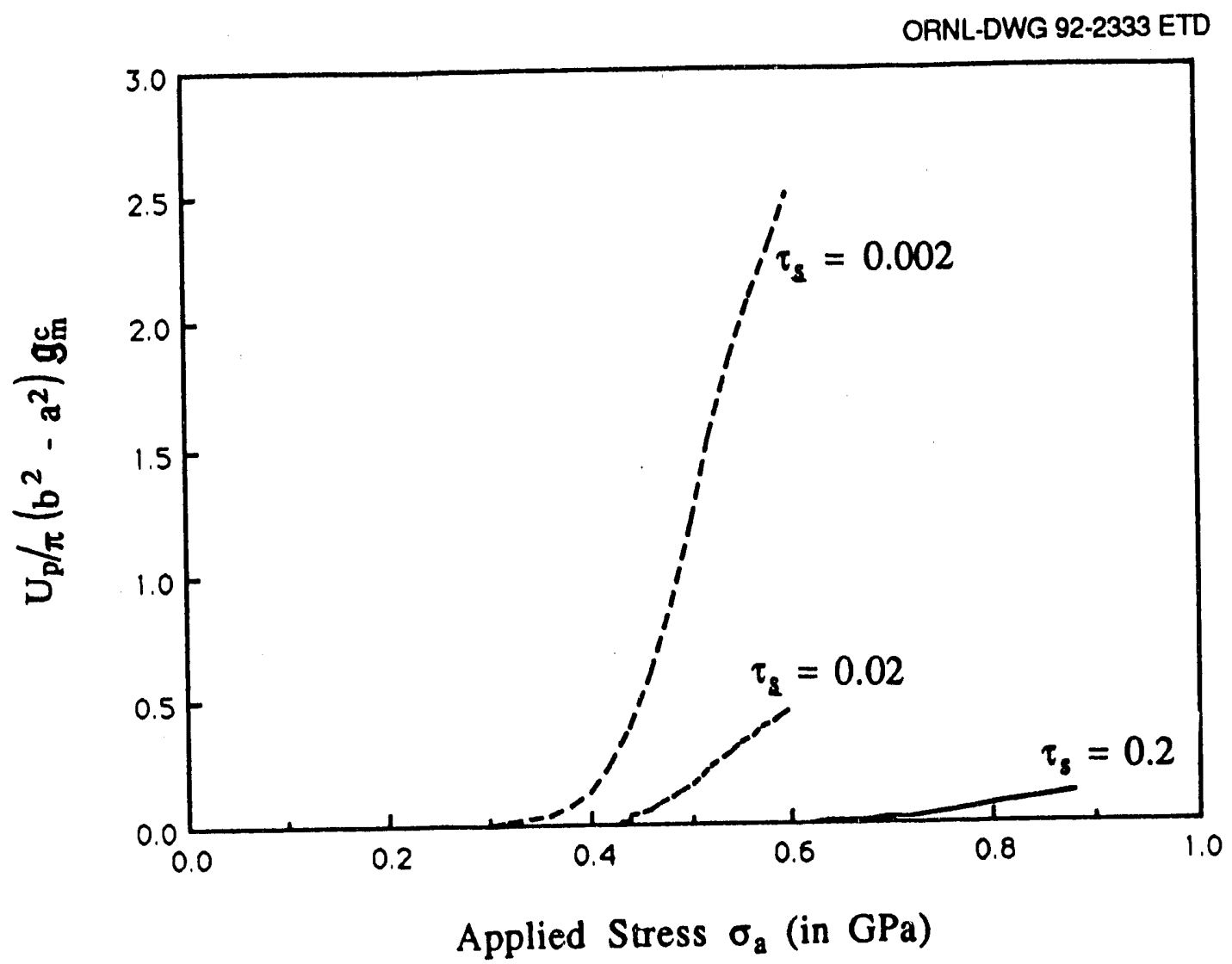

Fig. 6.3. The portion of pull-out energy $U_{p}$ [Eq. (53)], nondimensionalized by matrix fracture energy, vs the applied stress, $\sigma_{a}$, for several values of interfacial shear strength, $\tau_{s}$ (in GPa). Case of $\alpha_{f}=\alpha_{m}$. All plots were terminated at $P_{f}=1.0$. 


\section{CONCLUSIONS}

The results presented in this work illuminate some aspects of the complex response of fiberreinforced ceramic composites. Figures 4.2 through 4.5 exhibit the effects of various individual parameters, such as the interfacial strength $\tau_{s}$, the matrix fracture toughness $\mathfrak{g}_{m}^{c}$, and the thermal mismatch $\left(\alpha_{m}-\alpha_{f}\right)$, on the stress-strain response of the composite as attributed to progressive matrix cracking alone.

The failure of fibers is predicted by means of Weibull statistics; Figs. 5.1 and 5.2 show the relations between fiber failure probabilities, under monotonically increasing load, and some of the above-mentioned material parameters. The high sensitivity of the latter probabilities to radial distributions of fiber stresses is shown in Figs. 5.3a and 5.3b. This sensitivity is amplified because the "Weibull modulus" $M$ for ceramic fibers has rather high values, namely $8<M<10$. As can be noted from Figs. 5.1 to 5.3, the probabilities of fiber failure remain very small at low load level and take sharp upswings in the neighborhoods of some characteristic loads. It was found that the conditional probability of fiber failures to occur within any of the slip zones near the matrix cracks does not differ substantially from the probability of fiber failure anywhere along its length. Consequently, in all realistic cases, the phenomenon of fiber pull-out can be associated with fiber failures taking place within the slip zones. A certain portion of the irreversible fiber-fracture energy, $U_{p}$, which is surmised to be the major part of the energy dissipated within an RVE due to fiber break, is exhibited in Fig. 6.3 vs the externally applied stress $\sigma_{a}$ for various values of $\tau_{s}$. It is seen that $U_{p}$ is much larger for weak interfaces than for strong interfaces. However, the combination of matrix crack and a fiber failure within the adjacent slip zone brings about the local failure of the RVE and the overloading of neighboring fibers. This transfer of load beyond the region of a single RVE requires a separate analysis.

It would seem from Fig. 4.2 that an increase in the interfacial strength $\tau_{s}$ may lead to a better composite. However, such an inference is contradicted by the results shown in Figs. 5.1 and 6.3. When Figs. 4.2, 5.1, and 6.3 are viewed together, it can be seen that fiber strengths are likely to be exhausted before matrix cracking at higher values of $\tau_{s}$ and material failure would occur in a brittle manner. On the other hand, sufficiently low levels of $\tau_{s}$ will give rise to matrix cracks, fiber failures, and fiber pull-outs, resulting in departures from linear stress-strain behavior before ultimate failure.

Finally, bear in mind that at elevated temperatures detrimental effects of oxidation may bring about a brittle-like response of the ceramic composite. As can be seen from Figs. 4.7 and 4.8 , smaller values of $\tau_{s}$ cause a larger profusion of matrix cracks, which expose the composite to faster oxidation. This factor must be considered when selecting a specific interfacial strength among several available options. 


\section{REFERENCES}

Aveston, J., Cooper, G.A., and Kelly, A. November 1971. "Single and Multiple Fracture," pp. 15-26 in The Properties of Fibre Composites, Conference Proceedings, National Physical Laboratory, IPC Science and Technology Press Ltd.

Bogy, D.B. 1968. "Edge-Bonded Dissimilar Orthogonal Elastic Wedges Under Normal and Shear Loading," J. Appl. Mech. 35, 460.

Budiansky, B., Hutchinson, J.W., and Evans, A.G. 1986. "Matrix Fracture in Fiber-Reinforced Ceramics," J. Mech. Phys. Solids 34 (2), 167-189.

Daniel, I.M., Anastassopoulos, G., and Lee, J.-W. 1989. "Experimental Micromechanics of Brittle-Matrix Composites," pp. 133-146 in Micromechanics: Experimental Techniques, ed. W.N. Sharpe, Jr., ASME Publication AMD, 102.

Dollar, A., and Steif, P. S. 1988. "Load Transfer in Composites with a Coulomb Friction Interface," Int. J. Solids Struct. 24 (8), 789-803.

Dollar, A., and Steif, P. S. June 1989. "A Tension Crack Impinging Upon Frictional Interfaces," J. Appl. Mech. 56, 291-298.

Evans, A.G. 1990. "Perspective on the Development of High-Toughness Ceramics," J. Am. Ceram. Soc. 73 (2), 187-206.

Fang, G.P., Schapery, R.A., and Weitsman, Y. 1989. "Thermally-Induced Fracture in Composites," Eng. Fract. Mech. 33 (4), 619-632.

Hillig, W.B. 1987. "Strength and Toughness of Ceramic Matrix Composites," Am. Rev. Mater. Sci. 17, 341-383.

Hutchinson, J.W., and Jensen, H.M. 1990. "Models of Fiber Debonding and Pullout in Brittle Composites with Friction," Mech. Matcr. 9, Elsevier, 139-163.

Kelly, A., and Macmillan, N.H. 1986. Strong Solids, 3rd ed., Clarendon Press, Oxford, pp. 278.

Kimber, A.C., and Keer, J.G. 1982. "On the "Theoretical Average Crack Spacing in Brittle Matrix Composites Containing Continuous Aligned Fibres," J. Mater. Sci. Lett. 1, 353-354.

McCartney, L.N. 1989. "New Theoretical Model of Stress Transfer Between Fibre and Matrix in a Uniaxially Fibre-Reinforced Composite," pp. 215-244 in Proceedings of the Royal Society of London, Series A 425.

Nardone, V.C., and Prewo, K.M. 1988. "Tensile Performance of Carbon-Fibre-Reinforced Glass," J. Mater. Sci. 23, 168-180.

Nuismer, R.J., and Tan, S.C. 1988. "Constituti' e Relations of a Cracked Composite Lamina," J. Compos. Mater. 22, 306-340.

Schioler, L.J., and Stiglich, J.J., Jr. 1986. "Ceramic Matrix Composites: A Literature Review," Ceram. Bull. 65(2), 289-292. 
Schwietert, H.R., and Steif, P.S. 1990. "A Theory for the Ultimate Strength of a Brittle-Matrix Composite," J. Mech. Phys. Solids 38(3), 325--343.

Steif, P.S., and Dollar, A. 1988. "Longitudinal Shearing of a Weakly Bonded Fiber Composite," J. Appl. Mech. 55, 618-623.

Sutcu, M. 1989. "Weibull Statistics Applied to Fiber Failure in Ceramic Composites and Work of Fracture," Acta Metall. 37(2), 651-661.

Tan, S.C., and Nuismer, R.J. 1989. "A Theory for Progressive Matrix Cracking in Composite Laminates," J. Compos. Mater. 23, 1029-1047.

Thouless, M.D., and Evans, A.G. 1988. "Effects of Pull-Out on the Mechanical Properties of Ceramic-Matrix Composites," Acta Metall. 36(3), 517-522.

Timoshenko, S., and Goodier, J.N. 1951. Theory of Elasticity, 2nd ed., McGraw-Hill Book Co., Inc., New York, 67.

Washizu, K. 1975. Variational Methods in Elasticity and Plasticity, 2nd ed., Pergamon Press, $27-29$.

Wijeyewickrema, A.C., et al. 1990. "The Annular Crack Surrounding an Elastic Fiber in a Tension Field," Int. J. Solids Struct. (to be published). 


\section{APPENDIX A}

\section{FORMULATION OF IN-PLANE FIELDS}

Consider the following kinematically admissible displacements fields expressed in cylindrical coordinates:

$$
\text { In the fiber: } w=\varepsilon_{0} z, \quad u=A r, \quad v=0 \text {; }
$$

$$
\text { In the matrix: } w=\varepsilon_{0} z, \quad u=A r+B\left(r-\frac{a}{r}\right), v=0 .
$$

Consequently, in the fiber:

$$
\begin{gathered}
\varepsilon_{z}^{f}=\varepsilon_{0}, \\
\varepsilon_{\theta}^{f}=\varepsilon_{r}^{f}=A ;
\end{gathered}
$$

and in the matrix:

$$
\begin{gathered}
\varepsilon_{z}^{m}=\varepsilon_{0}, \\
\varepsilon_{r}^{m}=A+B\left(1+\frac{a^{2}}{r^{2}}\right), \text { and } \\
\varepsilon_{\theta}^{m}=A+B\left(1-\frac{a^{2}}{r^{2}}\right),
\end{gathered}
$$

where $A, B$, and $\varepsilon_{0}$ are constants.

By the Hooke's law, the axial stresses in the fiber and the matrix are

$$
\begin{gathered}
\sigma_{z}^{f}(r, z)=\frac{E_{f}\left(1-v_{f}\right) \varepsilon_{0}}{\left(1+v_{f}\right)\left(1-2 v_{f}\right)}+\frac{2 E_{f} v_{f} A}{\left(1+v_{f}\right)\left(1-2 v_{f}\right)}-\frac{E_{f}}{\left(1-2 v_{f}\right)} \varepsilon^{f, t h}, \\
\sigma_{z}^{m}(r, z)=\frac{E_{m}\left(1-v_{m}\right) \varepsilon_{0}}{\left(1+v_{m}\right)\left(1-2 v_{m}\right)}+\frac{2 E_{m} v_{m}(A+B)}{\left(1+v_{m}\right)\left(1-2 v_{m}\right)}-\frac{E_{m}}{\left(1-2 v_{m}\right)} \varepsilon^{m, t h},
\end{gathered}
$$

where

$$
\varepsilon^{f, t h}=\alpha_{f} T \text { and } \varepsilon^{m, t h}=\alpha_{m} T .
$$

The force balance along $z$-direction provides 


$$
\begin{aligned}
\sigma_{a} & =\varepsilon_{0} E_{c}+\left(V_{f} E_{f}^{*} \frac{2 v_{f}}{1-v_{f}}+V_{m} E_{m}^{*} \frac{2 v_{m}}{1-v_{m}}\right) A+V_{m} E_{m}^{*} \frac{2 v_{m} B}{1-v_{m}} \\
& -\frac{1+v_{f}}{1-v_{f}} V_{f} E_{f}^{*} \varepsilon^{f, t h}-\frac{1+v_{m}}{1-v_{m}} V_{m} E_{m}^{*} \varepsilon^{m, t h}
\end{aligned}
$$

where

$$
\begin{gathered}
E_{c}=V_{f} E_{f}^{*}+V_{m} E_{m}^{*}, \\
E_{f}^{*}=\frac{E_{f}\left(1-v_{f}\right)}{\left(1+v_{f}\right)\left(1-2 v_{f}\right)}, \\
E_{m}^{*}=\frac{E_{m}\left(1-v_{m}\right)}{\left(1+v_{m}\right)\left(1-2 v_{m}\right)} .
\end{gathered}
$$

It follows that $\varepsilon_{0}$ in Eq. (A.11) is given by

$$
\begin{aligned}
\varepsilon_{0} & =\left[\sigma_{a}-\left(V_{f} E_{f}^{*} \frac{2 v_{f}}{1-v_{f}}+V_{m} E_{m}^{*} \frac{2 v_{m}}{1-v_{m}}\right) A-V_{m} E_{m}^{*} \frac{2 v_{m} B}{1-v_{m}}\right. \\
& \left.+\frac{1+v_{f}}{1-v_{f}} V_{f} E_{f}^{*} \varepsilon^{f, t h}+\frac{1+v_{m}}{1-v_{m}} V_{m} E_{m}^{*} \varepsilon^{m, t h}\right] / E_{c} .
\end{aligned}
$$

Turning to the radial stresses $\sigma_{r}$ in the fiber and matrix, we have

$$
\sigma_{r}^{f}=\frac{E_{f}^{*}}{1-v_{f}} A+\frac{E_{f}^{*} v_{f}}{1-v_{f}} \varepsilon_{0}-\frac{1+v_{f}}{1-v_{f}} E_{f}^{*} \varepsilon^{f, t h}
$$

and

$$
\sigma_{r}^{m}=\frac{E_{m}^{*}}{1-v_{m}}(A+B)+\frac{E_{m}^{*}\left(1-2 v_{m}\right) a^{2} B}{\left(1-v_{m}\right) r^{2}}+\frac{E_{m}^{*} v_{m}}{1-v_{m}} \varepsilon_{0}-\frac{1+v_{m}}{1-v_{m}} E_{m}^{*} \varepsilon^{m, t h}
$$

The continuity condition $\sigma_{r}^{f}=\sigma_{r}^{m}$ at $r=a$ and the boundary condition $\sigma_{r}^{m}=0$ at $r=b$ yield the following equations for the unknowns $A$ and $B$ :

$$
C_{11} A+C_{12} B=f_{1}
$$


49

$$
C_{21} A+C_{22} B=f_{2}
$$

where

$$
\begin{aligned}
& C_{11}=1-\left(v_{f} E_{f}^{*} \frac{2 v_{f}}{1-v_{f}}+V_{m} E_{m}^{*} \frac{2 v_{m}}{1-v_{m}}\right) \frac{v_{m}}{E_{c}} \\
& C_{12}=1+V_{f}\left(1-2 v_{m}\right)-\frac{2 v m^{2} V_{m} E_{m}^{*}}{\left(1-v_{m}\right) E_{c}} \\
& C_{21}=\left[1-\left(v_{f} E_{f}^{*} \frac{2 v_{f}}{1-v_{f}}+V_{m} E_{m}^{*} \frac{2 v_{m}}{1-v_{m}}\right) \frac{v_{f}}{E_{c}}\right] \frac{E_{f}^{*}}{1-v_{f}} \\
& -\left[1-\left(v_{f} E_{f}^{*} \frac{2 v_{f}}{1-v_{f}}+V_{m} E_{m}^{*} \frac{2 v_{m}}{1-v_{m}}\right) \frac{v_{m}}{E_{c}}\right] \frac{E_{m}^{*}}{1-v_{m}} \text {, } \\
& C_{22}=\frac{v_{m} V_{m} E_{m}^{*}}{\left(1-v_{m}\right) E_{c}}\left(\frac{E_{m}^{*} v_{m}}{1-v_{m}}-\frac{E_{f}^{*} v_{f}}{1-v_{f}}\right)-2 E_{m}^{*}, \\
& f_{1}=-\frac{v_{m} \sigma_{a}}{E_{c}}+\left(1+v_{m}\right) \varepsilon^{m, t h}-\frac{v_{m}}{E_{c}}\left(\frac{1+v_{f}}{1-v_{f}} V_{f} E_{f}^{*} \varepsilon^{f, t h}+\frac{1+v_{m}}{1-v_{m}} V_{m} E_{m}^{*} \varepsilon^{m, t h}\right), \\
& f_{2}=\frac{1+v_{f}}{1-v_{f}} E_{f}^{*} \varepsilon^{f, t h}-\frac{1+v_{m}}{1-v_{m}} E_{m}^{*} \varepsilon^{m, t h} \\
& +\left[\frac{v_{m} E_{m}^{*}}{\left(1-v_{m}\right) E_{c}}-\frac{v_{f} E_{f}^{*}}{\left(1-v_{f}\right) E_{c}}\right]\left(\sigma_{a}+\frac{1+v_{f}}{1-v_{f}} V_{f} E_{f}^{*} \varepsilon^{f, t h}+\frac{1+v_{m}}{1-v_{m}} V_{m} E_{m}^{*} \varepsilon^{m, t h}\right) .
\end{aligned}
$$

In addition, it may be noted from Eqs. (A.18) to (A.20) that $A$ and $B$ depend linearly on $\sigma_{a}, \varepsilon^{f}$, th, and $\varepsilon^{m, t h}$, namely

$$
A\left(\sigma_{a}, \varepsilon^{f, t h}, \varepsilon^{m, t h}\right)=\sigma_{a} A_{1}+\varepsilon^{f, t h} A_{2}+\varepsilon^{m, t h} A_{3}
$$

with

$$
A_{1}=\mathrm{O}\left(v_{m}\right)+\mathrm{O}\left(v_{f}\right)
$$

and

$$
B\left(\sigma_{a}, \varepsilon^{f, t h}, \varepsilon^{m, t h}\right)=\sigma_{a} B_{1}+\varepsilon^{f, t h} B_{2}+\varepsilon^{m, t h} B_{3}
$$


with

$$
B_{1}=\mathrm{O}\left(\mathrm{v}_{m}\right)+\mathrm{O}\left(\mathrm{v}_{f}\right)
$$

Consequently, it can be concluded that contribution to $\sigma_{z}^{f}$ in Eq. (A.8) and $\sigma_{z}^{m}$ in Eq. (A.9) from the mechanical part of the transverse strains is of $O\left(v_{m}^{2}\right), O\left(v_{f}^{2}\right)$, or $O\left(v_{f} v_{m}\right)$. The small significance of this contribution would mitigate the effects of inaccuracies caused by our cmployment of boundary conditions (4a) and (4b) in constructing the present model. 


\section{APPENDIX B \\ THE BOUNDARY ELEMENT SCHEME}

Consider a two-dimensional representative volume element (RVE) in rectangular Cartesian coordinates as shown in Fig. B.1. The RVE consists of two linear isotropic elastic materials, loaded as shown in the figure. Assume perfect bond to exist at the interfaces $|x|=a$ between the two materials for $\left|\tau_{x z}\right|<\tau_{s}$, and let slip occur when $\left|\tau_{x z}\right|=\tau_{s}$ at those interfaces. Denote by $l_{s}$ the length of the slip zone.

The continuity of displacement and stress components at the nonslip interface $0 \leq z \leq L / 2-$ $l_{s}$ is expressed by

$$
\tau_{x z}^{f}=\tau_{x z}^{m}, \quad \sigma_{x}^{f}=\sigma_{x}^{m}, \quad u_{x}^{f}=u_{x}^{m}, \quad \text { and } u_{z}^{f}=u_{z}^{m}
$$

While at the slip interface $L / 2-l_{s} \leq z \leq L / 2$, the mechanical conditions are given by

$$
\tau_{x z}^{f}=-\tau_{s}, \quad \tau_{x z}^{m}=-\tau_{s}, \quad \sigma_{x}^{f}=\sigma_{x}^{m}, \quad \text { and } u_{x}^{f}=u_{x}^{m} .
$$

In addition, the continuity of $\tau_{x z}$ at the interface imposed the following relation

$$
\tau_{x z}(z, x=a)=-\tau_{s},
$$

as $z$ approaches $L / 2-l_{s}$ from the nonslip side.

The boundary element method was employed to solve for the stress and displacement fields. The resulting stress components $\sigma_{z}^{f}$ were plotted vs $x$ at several levels of $z$ in Fig. B.2.

Notice that the value of $l_{s}$ is unknown a priori. Therefore, a guess initial value of $l_{s}$ is first introduced into the boundary element method algorithm, leading to a certain solution which, in general, does not match the requirement (B.3). This value of $l_{s}$ was then adjusted iteratively, with corresonding values of $l_{s}$, until Eq. (B.3) was satisfied to within a desired accuracy.

For comparison, Fig. B. 2 also contains plots of the values of $\sigma_{z}^{f}$ computed by the method developed in this paper for the concentric cylindrical model shown in Fig. 2.1. Equivalent elastic properties, geometrical parameters, and loading conditions were employed for both cases shown in Fig. B.2. 


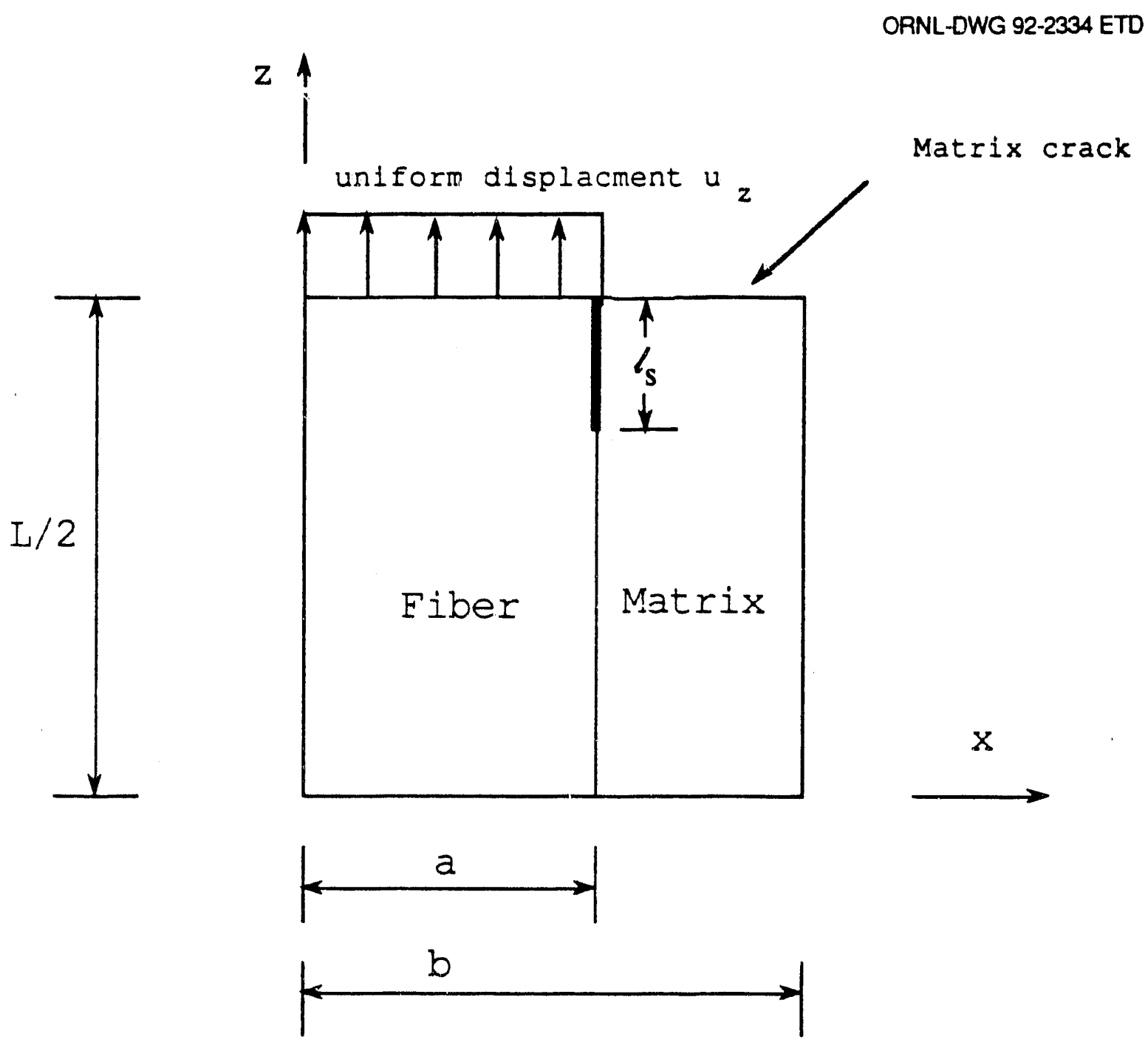

Fig. B.1. The Cartesian configuration employed for the boundary element method. The computations employed $L / a=8, a / b=0.4$. 


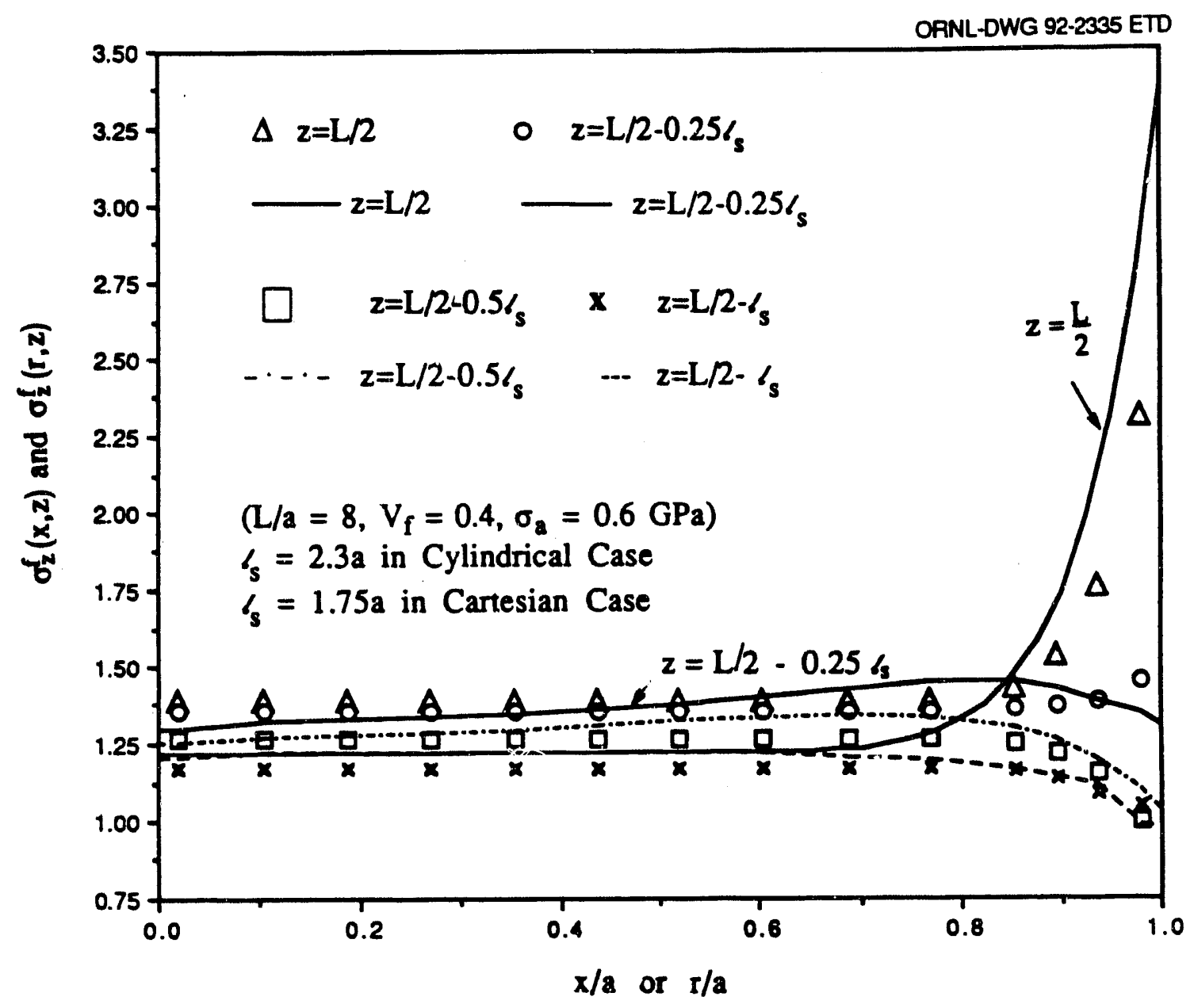

Fig. B.2. Profiles of fiber stresses $\sigma_{z}^{f}(z, z)$ vs $x / a$ computed by the boundary element method (discrete points) compared with $\sigma_{z}^{f}(r, z)$ vs r/a evaluated by the present model. 
ORNL-6703

\section{Internal Distribution}
1. P. F. Becher
2. T. M. Besman
3. H. W. Blake
4. J. J. Blass
5. R. A. Bradley
6. R. D. Cheverton
7. J. A. Clinard
8. J. L. Cook
9. J. M. Corum
10. D. F. Craig
11. R. C. Gwaltney
12. W. R. Hendrich
13. C. Hsueh
14. R. L. Huddleston
15. J. E. Jones Jr.
16. C. R. Luttrell
17. W. R. Martin
18. J. G. Merkle
19. S. E. Moore
20. D. G. O'Connor
21. A. B. Poole
22. C. E. Pugh
23. M. B. Ruggles
24. W. K. Sartory
25. J. T. Shaffer
26. F. W. Swinson
27. B. B. Thomas
28. D. A. Waters
29-43. Y. J. Weitsman
44. E.W. Whitfield
45. G. T. Yahr
46. A. Zucker
47. ORNL Patent Section
48. Central Research Library
49. Document Reference Section
50-51. Laboratory Records Department
52. Laboratory Records (RC)

\section{External Distribution}

53. J. A. Boulet, Dept. of Engineering Science and Mechanics, Rm. 310, Perkins Hall, The University of Tennessee, Knoxville, TN 37996-2030

54. T. G. Carley, Dept. of Engineering Science and Mechanics, Rm. 310, Perkins Hall, The University of Tennessee, Knoxville, TN 37996-2030

55. R. D. Krieg, Dept. of Engineering Science and Mechanics, Rm. 310, Perkins Hall, The University of Tennessee, Knoxville, TN 37996-2030

56. J. D. Landes, Dept. of Engineering Science and Mechanics, Rm. 310, Perkins Hall, The University of Tennessee, Knoxville, TN 37996-2030

57. M. Madhukar, Dept. of Engineering Science and Mechanics, Rm. 310, Perkins Hall, The University of Tennessee, Knoxville, TN 37996-2030

58. J. E. Stoneking, Dept. of Engineering Science and Mechanics, Rm. 310, Perkins Hall, The University of Tennessee, Knoxville, TN 37996-2030

59-61. H. Zhu, Dept. of Engineering Science and Mechanics, Rm. 310, Perkins Hall, The University of Tennessee, Knoxville, TN 37996-2030

62. J. D. Achenbach, Northwestem University, Catalysis Bldg., 2137 N Sheridan Rd., Evanston, IL 60208

63. D. Allen, Director, Ctr. for Mech. of Comp., Aer. Eng. Dept., Texas A\&M University, Room 736D-HR Bright Bldg., College Station, TX 77843-3141

64. G. L Anderson, Chief, Struct. and Dynamics Branch, Engineering Sciences Div., U.S. Army Research Office, P.O. Box 12211, Research Triangle Park, NC 27709-2211

65. Professeur Agrege, Didier Baptiste, Ecole Centrale Paris, Grande Voie des Vignes, 92295 Chatenay-Malabry cedex

66. S. N. Atluri, Georgia Institute of Technology, School of Eng. and Mech., Allanta, GA 30332

67. Jonathon Awerbuch, Drexel University, Mechnical Engineering Department, Philadelphia, PA 19104 
68. B. Budiansky, Division of Applied Sciences, Harvard University, Cambridge, MA 02138

69. C. C. Chamis, National Aeronautics and Space Administration, Lewis Rescarch Center, Cleveland OH 44135

70. F. P. Chiang, State University of New York, Department of Mechanical Engineering, Stony Brook, NY 11794

71. Tsu-Wei Chou, University of Delaware, Department of Mechanical Engineering, Newark, DE 19711

72. Francois Christin, Mat. Devt. Mgr., Comp. Operations, Societe Europenne de Propulsion, Solid Propulsion and Composites Division, Le Haillan B.P. 37, 33165 St. Medard en Jalles France

73. R. M. Christensen, Fiber Composites and Mechanics, Lawrence Livermore National Laboratory, L-338, University of California, P.O. Box 808, Livermore, CA 94550

74. I. M. Daniel, Robert R. McCormick School of Engineering and Applied Sciences, Northwestern University, Evanston, IL 60208

75. F. Delale, Dept. of Mechnical Engineering, The City College, Convent Ave. at 183th St., New York, NY 10031

76. D. C. Drucker, Division of Engineering Sciences and Mechanics, University of Florida, Gainesville, FL 32611

77. G. J. Dvorak, Head, Civil Engineering Department, Rensselaer Polytechnic Institute, Troy, NY 12181

78. W. W. Feng, Lawrence Livermore National Laboratory, L-342, Livermore, CA 94550

79. L. B. Freund, Division of Engineering, Brown University, Providence, RI 02912

80. R. H. Gallagher, University of Arizona, Dean of Engineering, Tucson, AZ 85721

81. J. G. Goree, Clemson University, 320 Riggs Hall, Clemson, SC 29634-0921

82. Tom Hahn, Department of Engineering Sciences and Mechanics, Pennsylvania State University, 227 Hammond Bldg., University Park, PA 16802

83. P. G. Hodge, Jr., Department of Aerospace Engineering and Mechanics, University of Minnesota, Minneapolis, MN 55455

84. W. F. Jones, Program Manager, Directorate of Aero. Sciences, AFOSR/NA, Boling Air Force Base. Washington, DC 20332-6448

85. Nils Juhlin, VP, NovaComp Eng., Inc., P.O. Box 95137, Seattle, WA 98145-2137

86. M. F. Kanninen, Southwest Research Institute, P.O. Drawer 28510, 6220 Culebra Rd., San Antonio, TX 78284

87. Keith Kedward, University of Califomia, Department of Mechinical Engineering, Santa Barbara, CA 93106

88. Prof. Kinra, Mech. and Mat. Eng., Texas A\&M Unive:sity, College Station, TX 77843

89. A. S. Kobayashi, Department of Mechanical Engineering, University of Washington, Seattle, WA 98195

90. Dusan Krajcinovic, Department of Mech. and Aero. Eng., Arizona State University, Tempe, AZ 85287-6106

91. F. A. Leckie, Head, Department of Mechanical Engineering, University of California, Santa Barbara, Santa Barbara, CA 93106

92. Norman Laws, Department of Mechnical Engineering, University of Pittsburgh, Pittsburgh, PA 15260

93. Harold Liebowitz, Dean, School of Eng. and Appl. Sci., George Washington University, Washington, DC 20052

94. L. N. McCartney, National Physical Laboratory, Div. of Materials Metrology, Bldg. 13, Teddington, Middlesix TW11 0LW, England

95. F. A. McClintock, Department of Mechanical Engineering, Massachusetts Institute of Technology, Cambridge, MA 02139

96. Hidenori Murakami, University of California, Department of AMES, B-010, LaJolla, CA 92093

97. Seiichi Nomura, Assoc. Prof. of Mechanical Engineering, The University of Texas, Box 19023, Arlington, TX 76019-0023

98. Ralph Nuismer, Hercules Corporation, P.O. Box 98, Magna, UT 84044 
99. Nicholas Pagano, Wright Laboratory MLBM, Wright Patterson Air Force Base, OH 45433

100. D. M. Parks, Massachusetts Institute of Technology, Department of Mech. Eng., Cambridge, MA 02139

101. Lee Phoenix, Sibley School of Mech. and Aero. Eng., Cornell University, Grumman and Upson Halls, Ithaca, NY 14853-7501

102. Daniel Post, Virginia Ploytechnic Institute, Dept. of Eng. Sci. and Mech., Blacksburg, VA 24061

103. Ryszard Pyrz, Aalborg University, Inst. of Mech. Eng., Pontoppidanstrabe 101, DK-9220 Aalborg, Denmark

104. F. G. Rammerstorfer, Christian Doppler Lab., Micromech of Materials, Gusshausstrasse 27-29, A-1040 Vienna, Austria

105. J. N. Reddy, Virginia Polytechnic Institute, Dept. of Eng. Sci. and Mech., 220 Norris Hall, Blacksburg, VA 24061

106. K. L. Reifsnider, Virginia Polytechnic Institute, Dept. of Eng. Sci. and Mech., Blacksburg, VA 24061

107. J. R. Rice, Harvard University, Div. of Appl. Sci., Cambridge, MA 02138

108. J. Rose, Drexel University, Dept. of Mech. Eng. and Mech., Philadelphia, PA 19104

109. A. A. Rubinstein, Tulane University, Dept. of Mech. Eng., New Orleans, LA 70118

110. J. L. Sanders, Harvard University, Div. of Appl. Sci., Cambridge, MA 02138

111. M. H. Santare, University of Delaware, Newark, DE 19716

112. R. A. Schapery, Aerospace Eng. and Eng. Mech., University of Texas, Austin, TX 78712-1085

113. G. C. M. Sih, LeHigh University, Inst. of Fracture and Solid Mechanics, Bethlehem, PA 18015

114. M. G. Simpson, Sr. Process Engineer, 3M Delta G, 9960-A Glenoaks Blvd., Sun Valley, CA 91352

115. Patrick Spriet, Applications Development Mgr., SEP, Inc., 1100 17th St. NW Suite 320, Washington, DC 20036

116. J. H. Starnes, Jr., NASA Langley Research Center, 8 W Taylor Rd., MS 190, Hampton, VA 23665

117. W. S. Steffier, Prog. Mgr., Ceramic-Matrix Composites, BP Chemicals Eng. Systems, 13722 Harvard Pl, Gardena, CA 90249-2529

118. C. T. Sun, Dept. of Aero. and Astro., Purdue University, Rm. 331, Grissom Hall, Lafayette, IN 47907

119. S. R. Swanson, Dept. of Mech. and Ind. Eng., University of Utah, Salt Lake City, UT 84112

120. Ramesh Talreja, Georgia Institute of Technology, School of Aero. Eng., Atlanta, GA 30332-0150

121. M. Taya, Dept. of Mechanical Engineering, University of Washington, Seattle, WA 98195

122. Prof. Walton, Dept. of Mech. Eng., Texas A\&M University, College Station, TX 77843

123. Albert Wang, Mech. Eng. Dept., Drexel University, Philadelphia, PA 19104

124. Su Su Wang, Dept. of Mech. Eng., University of Houston, 4800 Calhoun Rd., Houston, TX $77204-4792$

125. J. B. Whiteside, Head, Applied Mechanics, Research Department, Grumman Aerospace Corp., MS A08-35, Bethpage, NY 11714

126. S. W. Yurgartis, Dept. of Mech. and Aero. Eng., Clarkson University, Potsdam, NY $13699-5725$

127. Dr. Yevgeny, Eng., Prod. Design and Mech. Div., Alcoa Labs, ALCOA, Alcoa Center, PA 15069

128. Office of Assistant Manager for Energy Research and Development, Department of Energy, OR, Oak Ridge, TN 37831

129-138. Office of Scientific and Technical Information, P.O. Box 62, Oak Ridge, TN 37831 

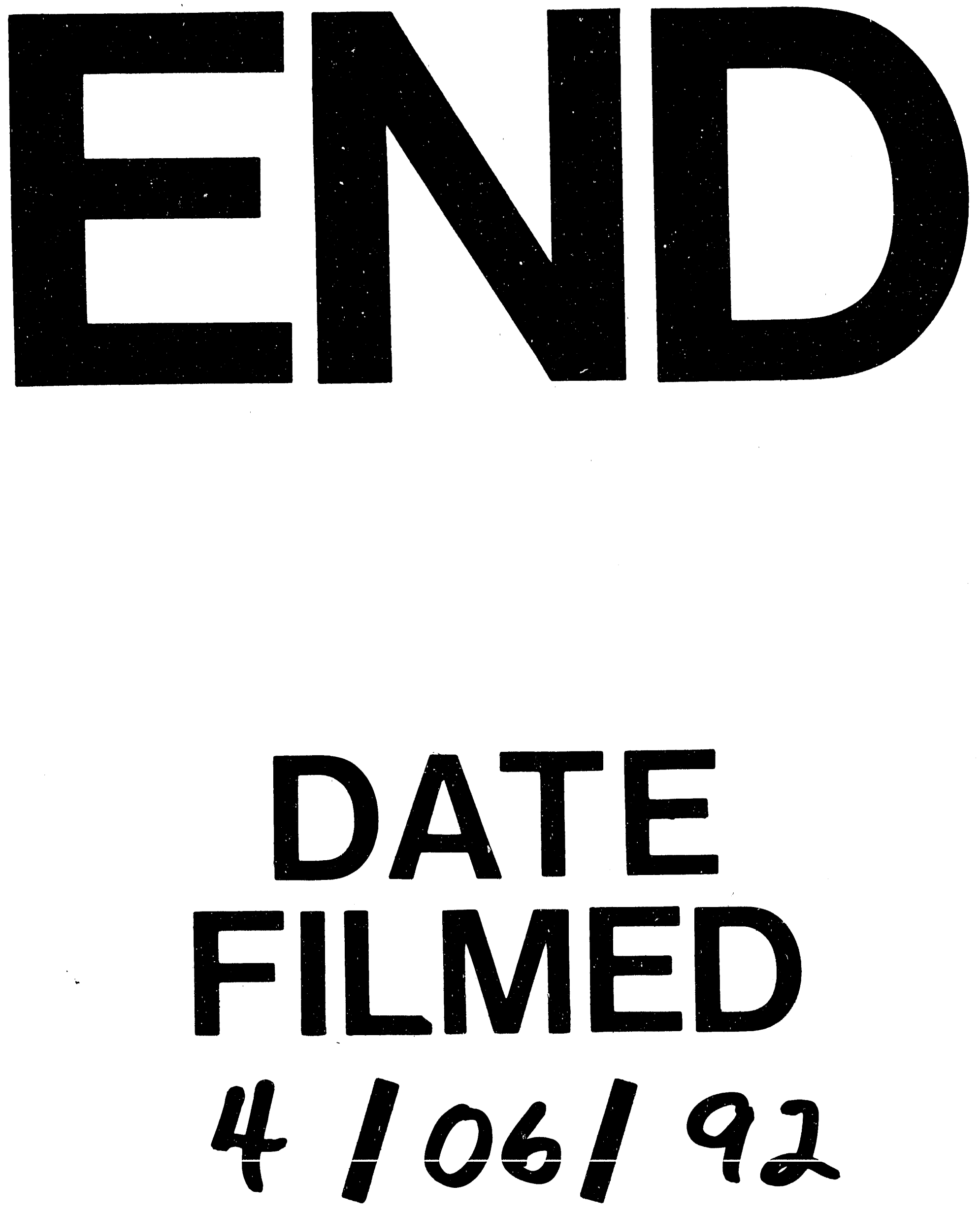
